\title{
Local well-posedness of a quasi-incompressible two-phase flow
}

\author{
Helmut ABELS AND JOSEF WEBER
}

\section{Dedicated to Matthias Hieber on the occasion of his 60th birthday}

Abstract. We show well-posedness of a diffuse interface model for a two-phase flow of two viscous incompressible fluids with different densities locally in time. The model leads to an inhomogeneous NavierStokes/Cahn-Hilliard system with a solenoidal velocity field for the mixture, but a variable density of the fluid mixture in the Navier-Stokes type equation. We prove existence of strong solutions locally in time with the aid of a suitable linearization and a contraction mapping argument. To this end, we show maximal $L^{2}$-regularity for the Stokes part of the linearized system and use maximal $L^{p}$-regularity for the linearized Cahn-Hilliard system.

\section{Introduction and main result}

In this contribution, we study a thermodynamically consistent, diffuse interface model for two-phase flows of two viscous incompressible system with different densities in a bounded domain in two or three space dimensions. The model was derived by Garcke and Grün [6] and leads to the following inhomogeneous Navier-Stokes/CahnHilliard system:

$$
\begin{aligned}
& \begin{aligned}
\partial_{t}(\rho \mathbf{v})+ & \operatorname{div}(\rho \mathbf{v} \otimes \mathbf{v})+\operatorname{div}\left(\mathbf{v} \otimes \frac{\tilde{\rho}_{1}-\tilde{\rho}_{2}}{2} m(\varphi) \nabla\left(\frac{1}{\varepsilon} W^{\prime}(\varphi)-\varepsilon \Delta \varphi\right)\right) \\
& =\operatorname{div}(-\varepsilon \nabla \varphi \otimes \nabla \varphi)+\operatorname{div}(2 \eta(\varphi) D \mathbf{v})-\nabla q,
\end{aligned} \\
& \operatorname{div} \mathbf{v}=0, \\
& \partial_{t} \varphi+\mathbf{v} \cdot \nabla \varphi=\operatorname{div}(m(\varphi) \nabla \mu), \\
& \mu=-\varepsilon \Delta \varphi+\frac{1}{\varepsilon} W^{\prime}(\varphi)
\end{aligned}
$$

Mathematics Subject Classification: 76T99, 35Q30, 35Q35, 76D03, 76D05, 76D27, 76D45

Keywords: Two-phase flow, Navier-Stokes equation, Diffuse interface model, Mixtures of viscous fluids, Cahn-Hilliard equation.

The authors acknowledge support by the SPP 1506 "Transport Processes at Fluidic Interfaces" of the German Science Foundation (DFG) through Grant GA695/6-1 and GA695/6-2. The results are part of the second author's PhD-thesis [16]. 
in $Q_{T}:=\Omega \times(0, T)$ together with the initial and boundary values

$$
\begin{aligned}
& \mathbf{v}_{\mid \partial \Omega}=\partial_{n} \varphi_{\mid \partial \Omega}=\partial_{n} \mu_{\mid \partial \Omega}=0 \quad \text { on }(0, T) \times \partial \Omega, \\
& \varphi(0)=\varphi_{0}, \mathbf{v}(0)=\mathbf{v}_{0} \quad \text { in } \Omega .
\end{aligned}
$$

Here, $\Omega \subseteq \mathbb{R}^{d}, d=2,3$, is a bounded domain with $C^{4}$-boundary. In this model, the fluids are assumed to be partly miscible and $\varphi: \Omega \times(0, T) \rightarrow \mathbb{R}$ denotes the volume fraction difference of the fluids. $\mathbf{v}, q$, and $\rho$ denote the mean velocity, the pressure and the density of the fluid mixture. It is assumed that the density is a given function of $\varphi$, more precisely

$$
\rho=\rho(\varphi)=\frac{\tilde{\rho}_{1}+\tilde{\rho}_{2}}{2}+\frac{\tilde{\rho}_{2}-\tilde{\rho}_{1}}{2} \varphi \quad \text { for all } \varphi \in \mathbb{R} .
$$

where $\tilde{\rho}_{1}, \tilde{\rho}_{2}$ are the specific densities of the (non-mixed) fluids. Moreover, $\mu$ is a chemical potential and $W(\varphi)$ is a homogeneous free energy density associated with the fluid mixture, $\varepsilon>0$ is a constant related to "thickness" of the diffuse interface, which is described by $\{x \in \Omega:|\varphi(x, t)|<1-\delta\}$ for some (small) $\delta>0$, and $m(\varphi$ ) is a mobility coefficient, which controls the strength of the diffusion in the system. Finally, $\eta(\varphi)$ is a viscosity coefficient and $D \mathbf{v}=\frac{1}{2}\left(\nabla \mathbf{v}+\nabla \mathbf{v}^{T}\right)$.

Existence of weak solution for this system globally in time was shown by Depner and Garcke [4] and [5] for non-degenerate and degenerate mobility in the case of a singular free energy density $W$. Moreover, Grün [13] convergence (of suitable subsequences) of a fully discrete finite-element scheme for this system to a weak solution in the case of a smooth $W: \mathbb{R} \rightarrow \mathbb{R}$ with suitable polynomial growth. In the case of dynamic boundary conditions, which model moving contact lines, existence of weak solutions for this system was shown by Gal et al. [11]. In the case of non-Newtonian fluids of suitable $p$-growth, existence of weak solutions was proved by Abels and Breit [3]. For the case of a non-local Cahn-Hilliard equation and Newtonian fluids, the corresponding results was derived by Frigeri [10] and for a model with surfactants by Garcke and the authors in [7]. Recently, Giorgini [12] proved existence of local strong solutions in a two-dimensional bounded, sufficiently smooth domain and global existence of strong solutions in the case of a two-dimensional torus.

Remark 1. In [4], it is shown that the first equation is equivalent to

$$
\begin{aligned}
& \rho \partial_{t} \mathbf{v}+\left(\rho \mathbf{v}+\frac{\tilde{\rho}_{1}-\tilde{\rho}_{2}}{2} m(\varphi) \quad \nabla\left(\frac{1}{\varepsilon} W^{\prime}(\varphi)-\varepsilon \Delta \varphi\right)\right) \cdot \nabla \mathbf{v}+\nabla p-\operatorname{div}(2 \eta(\varphi) D \mathbf{v}) \\
& \quad=-\varepsilon \Delta \varphi \nabla \varphi
\end{aligned}
$$

This reformulation will be useful in our analysis.

For the following, we assume: 
Assumption 1. 1. Let $\Omega \subseteq \mathbb{R}^{d}$ be a bounded domain with $C^{4}$-boundary and $d=2,3$.

2. Let $\eta, m \in C_{b}^{5}(\mathbb{R})$ be such that $\eta(s) \geq \eta_{0}>0$ and $m(s) \geq m_{0}$ for every $s \in \mathbb{R}$ and some $\eta_{0}, m_{0}>0$.

3. The density $\rho: \mathbb{R} \rightarrow \mathbb{R}$ is given by

$$
\rho=\rho(\varphi)=\frac{\tilde{\rho}_{1}+\tilde{\rho}_{2}}{2}+\frac{\tilde{\rho}_{2}-\tilde{\rho}_{1}}{2} \varphi \quad \text { for all } \varphi \in \mathbb{R}
$$

4. $W: \mathbb{R} \rightarrow \mathbb{R}$ is five times continuously differentiable.

With these assumptions, we will show our main existence result on short-time existence of strong solutions for (1)-(6):

Theorem 2. (Existence of strong solutions) Let $\Omega, \eta, m, \rho$ and $W$ be as in Assumption 1. Moreover, let $\mathbf{v}_{0} \in H_{0}^{1}(\Omega)^{d} \cap L_{\sigma}^{2}(\Omega)$ and $\varphi_{0} \in\left(L^{p}(\Omega), W_{p, N}^{4}(\Omega)\right)_{1-\frac{1}{p}, p}$ be given with $\left|\varphi_{0}(x)\right| \leq 1$ for all $x \in \Omega$ and $4<p<6$. Then, there exists $T>0$ such that (1)-(6) has a unique strong solution

$$
\begin{aligned}
& \mathbf{v} \in W_{2}^{1}\left(0, T ; L_{\sigma}^{2}(\Omega)\right) \cap L^{2}\left(0, T ; H^{2}(\Omega)^{d} \cap H_{0}^{1}(\Omega)^{d}\right), \\
& \varphi \in W_{p}^{1}\left(0, T ; L^{p}(\Omega)\right) \cap L^{p}\left(0, T ; W_{p, N}^{4}(\Omega)\right),
\end{aligned}
$$

where $W_{p, N}^{4}(\Omega)=\left\{u \in W_{p}^{4}(\Omega):\left.\partial_{n} u\right|_{\partial \Omega}=\left.\partial_{n} \Delta u\right|_{\partial \Omega}=0\right\}$.

We will prove this result with the aid of a contraction mapping argument after a suitable reformulation, similar to [1]. But for the present system, the linearized system is rather different.

The structure of this contribution is as follows: in Sect. 2, we introduce some basic notation and recall some results used in the following. The main result is proved in Sect. 3. For its proof, we use suitable estimates of the nonlinear terms, which are shown in Sect. 4 , and a result on maximal $L^{2}$-regularity of a Stokes-type system, which is shown in Sect. 5.

\section{Preliminaries}

For an open set $U \subseteq \mathbb{R}^{d}, m \in \mathbb{N}_{0}$ and $1 \leq p \leq \infty$, we denote by $W_{p}^{m}(U)$ the $L^{p}$-Sobolev space of order $m$ and $W_{p}^{m}(U ; X)$ its $X$-valued variant, where $X$ is a Banach space. In particular, $L^{p}(U)=W_{p}^{0}(U)$ and $L^{p}(U ; X)=W_{p}^{0}(U ; X)$. Moreover, $B_{p q}^{s}(\Omega)$ denotes the standard Besov space, where $s \in \mathbb{R}, 1 \leq p, q \leq \infty$, and $L_{\sigma}^{2}(\Omega)$ is the closure of $C_{0, \sigma}^{\infty}(\Omega)=\left\{\mathbf{u} \in C_{0}^{\infty}(\Omega)^{d}: \operatorname{div} \mathbf{u}=0\right\}$ in $L^{2}(\Omega)^{d}$ and $\mathbb{P}_{\sigma}: L^{2}(\Omega)^{d} \rightarrow L_{\sigma}^{2}(\Omega)$ the orthogonal projection onto it, i.e., the Helmholtz projection.

We will frequently use: 
Theorem 3. (Composition with Sobolev functions) Let $\Omega \subseteq \mathbb{R}^{d}$ be a bounded domain with $C^{1}$-boundary, $m, n \in \mathbb{N}$ and let $1 \leq p<\infty$ such that $m-d p>0$. Then, for every $f \in C^{m}\left(\mathbb{R}^{N}\right)$ and every $R>0$ there exists a constant $C>0$ such that for all $u \in W_{p}^{m}(\Omega)^{N}$ with $\|u\|_{W_{p}^{m}(\Omega)^{N}} \leq R$, we have $f(u) \in W_{p}^{m}(\Omega)$ and $\|f(u)\|_{W_{p}^{m}(\Omega)} \leq$ C. Moreover, if $f \in C^{m+1}\left(\mathbb{R}^{N}\right)$, then for all $R>0$ there exists a constant $L>0$ such that

$$
\|f(u)-f(v)\|_{W_{p}^{m}(\Omega)} \leq L\|u-v\|_{W_{p}^{m}(\Omega)^{N}}
$$

for all $u, v \in W_{p}^{m}(\Omega)^{N}$ with $\|u\|_{W_{p}^{m}(\Omega)^{N}},\|v\|_{W_{p}^{m}(\Omega)^{N}} \leq R$.

Proof. The first part follows from [14, Chapter 5, Theorem 1 and Lemma]. The second part can be easily reduced to the first part.

In particular, we have $u v \in W_{p}^{m}(\Omega)$ for all $u, v \in W_{p}^{m}(\Omega)$ under the assumptions of the theorem.

Let $X_{0}, X_{1}$ be Banach spaces such that $X_{1} \hookrightarrow X_{0}$ densely. It is well known that

$$
W_{p}^{1}\left(I ; X_{0}\right) \cap L^{p}\left(I ; X_{1}\right) \hookrightarrow B U C\left(I ;\left(X_{0}, X_{1}\right)_{1-\frac{1}{p}, p}\right), \quad 1 \leq p<\infty,
$$

continuously for $I=[0, T], 0<T<\infty$, and $I=[0, \infty)$, cf. Amann [8, Chapter III, Theorem 4.10.2]. Here, $\left(X_{0}, X_{1}\right)_{\theta, p}$ denotes the real interpolation space of $\left(X_{0}, X_{1}\right)$ with exponent $\theta$ and summation index $p$. Moreover, $B U C(I ; X)$ is the space of all bounded and uniformly continuous $f: I \rightarrow X$ equipped with the supremum norm, where $X$ is a Banach space.

Moreover, we will use:

Lemma 1. Let $X_{0} \subseteq Y \subseteq X_{1}$ be Banach spaces such that

$$
\|x\|_{Y} \leq C\|x\|_{X_{0}}^{1-\theta}\|x\|_{X_{1}}^{\theta}
$$

for every $x \in X_{0}$ and a constant $C>0$, where $\theta \in(0,1)$. Then,

$$
C^{0, \alpha}\left([0, T] ; X_{1}\right) \cap L^{\infty}\left(0, T ; X_{0}\right) \hookrightarrow C^{0, \alpha \theta}([0, T] ; Y) .
$$

continuously.

The result is well-known and can be proved in a straight forward manner.

\section{Proof of the main result}

We prove the existence of a unique strong solution $(\mathbf{v}, \varphi) \in X_{T}$ for small $T>0$, where the space $X_{T}$ will be specified later. The idea for the proof is to linearize the highest order terms in the equations above at the initial data and then to split the equations in a linear and a nonlinear part such that

$$
\mathcal{L}(\mathbf{v}, \varphi)=\mathcal{F}(\mathbf{v}, \varphi)
$$


where we still have to specify in which sense this equation has to hold. To linearize it formally at the initial data, we replace $\mathbf{v}, p$ and $\varphi$ by $\mathbf{v}_{0}+\varepsilon \mathbf{v}, p_{0}+\varepsilon p$ and $\varphi_{0}+\varepsilon \varphi$ and then differentiate with respect to $\varepsilon$ at $\varepsilon=0$. In (1) and the equivalent equation (7), the highest order terms with respect to $t$ and $x$ are $\rho \partial_{t} \mathbf{v}, \operatorname{div}(2 \eta(\varphi) D \mathbf{v})$ and $\nabla p$. Hence, the linearizations are given by

$$
\begin{aligned}
\frac{d}{d \varepsilon}\left(\rho\left(\varphi_{0}+\varepsilon \varphi\right) \partial_{t}\left(\mathbf{v}_{0}+\varepsilon \mathbf{v}\right)\right)_{\mid \varepsilon=0} & =\rho^{\prime}\left(\varphi_{0}\right) \varphi \partial_{t} \mathbf{v}_{0}+\rho\left(\varphi_{0}\right) \partial_{t} \mathbf{v}=\rho_{0} \partial_{t} \mathbf{v}, \\
\frac{d}{d \varepsilon}\left(\operatorname{div}\left(2 \eta\left(\varphi_{0}+\varepsilon \varphi\right) D\left(\mathbf{v}_{0}+\varepsilon \mathbf{v}\right)\right)\right)_{\mid \varepsilon=0} & =\operatorname{div}\left(2 \eta^{\prime}\left(\varphi_{0}\right) \varphi D \mathbf{v}_{0}\right)+\operatorname{div}\left(2 \eta\left(\varphi_{0}\right) D \mathbf{v}\right), \\
\frac{d}{d \varepsilon} \nabla\left(p_{0}+\varepsilon p\right)_{\mid \varepsilon=0} & =\nabla p
\end{aligned}
$$

where $\rho_{0}:=\rho\left(\varphi_{0}\right)$ and $\rho_{0}^{\prime}:=\rho^{\prime}\left(\varphi_{0}\right)$. Moreover, we omit the term $\operatorname{div}\left(2 \eta^{\prime}\left(\varphi_{0}\right) \varphi D \mathbf{v}_{0}\right)$ in the second linearization since it is of lower order. For the last equation, we get the linearization

$$
\begin{aligned}
& \frac{\mathrm{d}}{\mathrm{d} \tilde{\varepsilon}} \operatorname{div}\left(m\left(\varphi_{0}+\tilde{\varepsilon} \varphi\right) \nabla\left(-\varepsilon \Delta\left(\varphi_{0}+\tilde{\varepsilon} \varphi\right)\right)\right)_{\mid \tilde{\varepsilon}=0} \\
& \quad=-\varepsilon \operatorname{div}\left(m^{\prime}\left(\varphi_{0}\right) \varphi \nabla \Delta \varphi_{0}\right)-\varepsilon \operatorname{div}\left(m\left(\varphi_{0}\right) \nabla \Delta \varphi\right) .
\end{aligned}
$$

We can omit the first term since it is of lower order. The second term can formally be reformulated as

$$
-\varepsilon \operatorname{div}\left(m\left(\varphi_{0}\right) \nabla \Delta \varphi\right)=-\varepsilon m^{\prime}\left(\varphi_{0}\right) \nabla \varphi_{0} \cdot \nabla \Delta \varphi-\varepsilon m\left(\varphi_{0}\right) \Delta(\Delta \varphi)
$$

Here, the first summand is of lower order again. Hence, the linearization is given by $-\varepsilon m\left(\varphi_{0}\right) \Delta^{2} \varphi$ upto terms of lower order. Due to these linearizations, we define the linear operator $\mathcal{L}: X_{T} \rightarrow Y_{T}$ by

$$
\mathcal{L}(\mathbf{v}, \varphi)=\left(\begin{array}{c}
\mathbb{P}_{\sigma}\left(\rho_{0} \partial_{t} \mathbf{v}\right)-\mathbb{P}_{\sigma}\left(\operatorname{div}\left(2 \eta\left(\varphi_{0}\right) D \mathbf{v}\right)\right) \\
\partial_{t} \varphi+\varepsilon m\left(\varphi_{0}\right) \Delta^{2} \varphi
\end{array}\right)
$$

where $\mathcal{L}$ consists of the principal part of the lionization's, i.e., of the terms of the highest order. Furthermore, we define the nonlinear operator $\mathcal{F}: X_{T} \rightarrow Y_{T}$ by

$$
\mathcal{F}(\mathbf{v}, \varphi)=\left(\begin{array}{c}
\mathbb{P}_{\sigma} F_{1}(\mathbf{v}, \varphi) \\
-\nabla \varphi \cdot \mathbf{v}+\operatorname{div}\left(\frac{1}{\varepsilon} m(\varphi) \nabla W^{\prime}(\varphi)\right)+\varepsilon m\left(\varphi_{0}\right) \Delta^{2} \varphi-\varepsilon \operatorname{div}(m(\varphi) \nabla \Delta \varphi)
\end{array}\right),
$$

where

$$
\begin{aligned}
F_{1}(\mathbf{v}, \varphi) & =\left(\rho_{0}-\rho\right) \partial_{t} \mathbf{v}-\operatorname{div}\left(2 \eta\left(\varphi_{0}\right) D \mathbf{v}\right)+\operatorname{div}(2 \eta(\varphi) D \mathbf{v})-\varepsilon \Delta \varphi \nabla \varphi \\
& -\left(\left(\rho \mathbf{v}+\frac{\tilde{\rho}_{1}-\tilde{\rho}_{2}}{2} m(\varphi) \nabla\left(\frac{1}{\varepsilon} W^{\prime}(\varphi)-\varepsilon \Delta \varphi\right)\right) \cdot \nabla\right) \mathbf{v}
\end{aligned}
$$


It still remains to define the spaces $X_{T}$ and $Y_{T}$. To this end, we set

$$
\begin{aligned}
& Z_{T}^{1}:=L^{2}\left(0, T ; H^{2}(\Omega)^{d} \cap H_{0}^{1}(\Omega)^{d}\right) \cap W_{2}^{1}\left(0, T ; L_{\sigma}^{2}(\Omega)\right), \\
& Z_{T}^{2}:=L^{p}\left(0, T ; W_{p, N}^{4}(\Omega)\right) \cap W_{p}^{1}\left(0, T ; L^{p}(\Omega)\right)
\end{aligned}
$$

with $4<p<6$, where

$$
W_{p, N}^{4}(\Omega):=\left\{\varphi \in W_{p}^{4}(\Omega) \mid \partial_{n} \varphi=\partial_{n}(\Delta \varphi)=0\right\} .
$$

We equip $Z_{T}^{1}$ and $Z_{T}^{2}$ with the norms $\|\cdot\|_{Z_{T}^{1}}^{\prime}$ and $\|\cdot\|_{Z_{T}^{2}}^{\prime}$ defined by

$$
\begin{aligned}
\|\mathbf{v}\|_{Z_{T}^{1}}^{\prime} & :=\left\|\mathbf{v}^{\prime}\right\|_{L^{2}\left(0, T ; L^{2}(\Omega)\right)}+\|\mathbf{v}\|_{L^{2}\left(0, T ; H^{2}(\Omega)\right)}+\|\mathbf{v}(0)\|_{\left(L^{2}(\Omega), H^{2}(\Omega)\right)_{\frac{1}{2}, 2}}, \\
\|\varphi\|_{Z_{T}^{2}}^{\prime} & :=\left\|\varphi^{\prime}\right\|_{L^{p}\left(0, T ; L^{p}(\Omega)\right)}+\|\varphi\|_{L^{p}\left(0, T ; W_{p, N}^{4}(\Omega)\right)}+\|\varphi(0)\|_{\left(L^{p}(\Omega), W_{p}^{4}(\Omega)\right)_{1-\frac{1}{p}, p}} .
\end{aligned}
$$

We use these norms since they guarantee that for all embeddings we will study later the embedding constant $C$ does not depend on $T$, cf. Lemma 2. To this end, we use:

Lemma 2. Let $0<T_{0}<\infty$ be given and $X_{0}, X_{1}$ be some Banach spaces such that $X_{1} \hookrightarrow X_{0}$ densely. For every $0<T<\frac{T_{0}}{2}$, we define

$$
X_{T}:=L^{p}\left(0, T ; X_{1}\right) \cap W_{p}^{1}\left(0, T ; X_{0}\right),
$$

where $1 \leq p<\infty$, equipped with the norm

$$
\|u\|_{X_{T}}:=\|u\|_{L^{p}\left(0, T ; X_{1}\right)}+\|u\|_{W_{p}^{1}\left(0, T ; X_{0}\right)}+\|u(0)\|_{\left(X_{0}, X_{1}\right)_{1-\frac{1}{p}, p}} .
$$

Then, there exists an extension operator $E: X_{T} \rightarrow X_{T_{0}}$ and some constant $C>0$ independent of $T$ such that $E u_{\mid(0, T)}=u$ in $X_{T}$ and

$$
\|E u\|_{X_{T_{0}}} \leq C\|u\|_{X_{T}}
$$

for every $u \in X_{T}$ and every $0<T<\frac{T_{0}}{2}$. Moreover, there exists a constant $\tilde{C}\left(T_{0}\right)>0$ independent of $T$ such that

$$
\|u\|_{B U C\left([0, T] ;\left(X_{0}, X_{1}\right)_{1-\frac{1}{p}, p}\right)} \leq \tilde{C}\left(T_{0}\right)\|u\|_{X_{T}}
$$

for every $u \in X_{T}$ and every $0<T<\frac{T_{0}}{2}$.

Proof. The result is well-known. In the case $u(0)=0$, one can prove the result with the aid of the extension operator defined by

$$
(E u)(t):= \begin{cases}u(t) & \text { if } t \in[0, T], \\ u(2 T-t) & \text { if } t \in(T, 2 T], \\ 0 & \text { if } t \in\left(2 T, T_{0}\right] .\end{cases}
$$

The case $u(0) \neq 0$ can be easily reduced to the case $u(0)=0$ by substracting a suitable extension of $u_{0}$ to $[0, \infty)$. We refer to [16, Lemma 5.2] for the details. 
The last preparation before we can start with the existence proof is the definition of the function spaces $X_{T}:=X_{T}^{1} \times X_{T}^{2}$ and $Y_{T}$ by

$$
\begin{aligned}
X_{T}^{1} & :=\left\{\mathbf{v} \in Z_{T}^{1} \mid \mathbf{v}_{\mid t=0}=\mathbf{v}_{0}\right\}, \\
X_{T}^{2} & :=\left\{\varphi \in Z_{T}^{2} \mid \varphi_{\mid t=0}=\varphi_{0}\right\}, \\
Y_{T} & :=Y_{T}^{1} \times Y_{T}^{2}:=L^{2}\left(0, T ; L_{\sigma}^{2}(\Omega)\right) \times L^{p}\left(0, T ; L^{p}(\Omega)\right),
\end{aligned}
$$

where

$$
\mathbf{v}_{0} \in\left(L_{\sigma}^{2}(\Omega), H^{2}(\Omega)^{d} \cap H_{0}^{1}(\Omega)^{d} \cap L_{\sigma}^{2}(\Omega)\right)_{\frac{1}{2}, 2}=H_{0}^{1}(\Omega)^{d} \cap L_{\sigma}^{2}(\Omega)
$$

and

$$
\varphi_{0} \in\left(L^{p}(\Omega), W_{p, N}^{4}(\Omega)\right)_{1-\frac{1}{p}, p}
$$

are the initial values from (6). Note that in the space $X_{T}^{2}$ we have to ensure that $\varphi_{\mid t=0}=\varphi_{0} \in[-1,1]$ since we will use this property to show the Lipschitz continuity of $\mathcal{F}: X_{T} \rightarrow Y_{T}$ in Proposition 1. Moreover, we note that $X_{T}$ is not a vector space due to the condition $\varphi_{\mid t=0}=\varphi_{0}$. It is only an affine linear subspace of $Z_{T}:=Z_{T}^{1} \times Z_{T}^{2}$.

Proposition 1. Let the Assumptions 1 hold and $\varphi_{0}$ be given as in Theorem 2. Then, there is a constant $C(T, R)>0$ such that

$$
\left\|\mathcal{F}\left(\mathbf{v}_{1}, \varphi_{1}\right)-\mathcal{F}\left(\mathbf{v}_{2}, \varphi_{2}\right)\right\|_{Y_{T}} \leq C(T, R)\left\|\left(\mathbf{v}_{1}-\mathbf{v}_{2}, \varphi_{1}-\varphi_{2}\right)\right\|_{X_{T}}
$$

for all $\left(\mathbf{v}_{i}, \varphi_{i}\right) \in X_{T}$ with $\left\|\left(\mathbf{v}_{i}, \varphi_{i}\right)\right\|_{X_{T}} \leq R$ and $i=1$, 2. Moreover, it holds $C(T, R) \rightarrow 0$ as $T \rightarrow 0$.

The proposition is proved in Sect. 4 below.

Theorem 4. Let $T>0$ and $\mathcal{L}, X_{T}$ and $Y_{T}$ be defined as before. Then, $\mathcal{L}: X_{T} \rightarrow Y_{T}$ is invertible. Moreover, for every $T_{0}>0$ there is a constant $C\left(T_{0}\right)>0$ such that

$$
\left\|\mathcal{L}^{-1}\right\|_{\mathcal{L}\left(Y_{T}, X_{T}\right)} \leq C\left(T_{0}\right) \quad \text { for all } T \in\left(0, T_{0}\right]
$$

This theorem is proved in Sect. 5 below.

Proof of Theorem 2. First of all we note that (1)-(4) is equivalent to

$$
(\mathbf{v}, \varphi)=\mathcal{L}^{-1}(\mathcal{F}(\mathbf{v}, \varphi)) \text { in } X_{T}
$$

The fact that $\mathcal{L}$ is invertible will be proven later. Equation (10) implies that we have rewritten the system to a fixed-point equation which we want to solve by using the Banach fixed-point theorem.

To this end, we consider some $(\tilde{\mathbf{v}}, \tilde{\varphi}) \in X_{T}$ and define

$$
M:=\left\|\mathcal{L}^{-1} \circ \mathcal{F}(\tilde{\mathbf{v}}, \tilde{\varphi})\right\|_{X_{T}}<\infty .
$$


Now, let $R>0$ be given such that $(\tilde{\mathbf{v}}, \tilde{\varphi}) \in \overline{B_{R}^{X_{T}}(0)}$ and $R>2 M$. Then, it follows from Proposition 1 that there exists a constant $C=C(T, R)>0$ such that

$$
\left\|\mathcal{F}\left(\mathbf{v}_{1}, \varphi_{1}\right)-\mathcal{F}\left(\mathbf{v}_{2}, \varphi_{2}\right)\right\|_{Y_{T}} \leq C(T, R)\left\|\left(\mathbf{v}_{1}, \varphi_{1}\right)-\left(\mathbf{v}_{2}, \varphi_{2}\right)\right\|_{X_{T}}
$$

for all $\left(\mathbf{v}_{i}, \varphi_{i}\right) \in X_{T}$ with $\left\|\left(\mathbf{v}_{i}, \varphi_{i}\right)\right\|_{X_{T}} \leq R, j=1,2$, where it holds $C(T, R) \rightarrow 0$ as $T \rightarrow 0$. Furthermore, we choose $T$ so small that

$$
\left\|\mathcal{L}^{-1}\right\|_{\mathcal{L}\left(Y_{T}, X_{T}\right)} C(T, R)<\frac{1}{2} .
$$

Here, we have to ensure that $\left\|\mathcal{L}^{-1}\right\|_{\mathcal{L}\left(Y_{T}, X_{T}\right)}$ does not converge to $+\infty$ as $T \rightarrow 0$. But, since Lemmas 7 and 9 below yield $\left\|\mathcal{L}^{-1}\right\|_{\mathcal{L}\left(Y_{T}, X_{T}\right)}<C\left(T_{0}\right)$ for every $0<T<T_{0}$ and for a constant that does not depend on $T$, this is not the case and we can choose $T>0$ in such a way that the previous estimate holds. Note that $T$ depends on $R$ and in general $T$ has to become smaller, the larger we choose $R$.

Since we want to apply the Banach fixed-point theorem on $\overline{B_{R}^{X_{T}}(0)} \subseteq X_{T}$ as we only consider functions $(\mathbf{v}, \varphi) \in X_{T}$ which satisfy $\|(\mathbf{v}, \varphi)\|_{X_{T}} \leq R$, we have to show that $\mathcal{L}^{-1} \circ \mathcal{F}$ maps from $\overline{B_{R}^{X_{T}}(0)}$ to $\overline{B_{R}^{X_{T}}(0)}$.

From the considerations above, we know that there exists $(\tilde{\mathbf{v}}, \tilde{\varphi}) \in \overline{B_{R}^{X_{T}}(0)}$ such that

$$
\left\|\mathcal{L}^{-1} \circ \mathcal{F}(\tilde{\mathbf{v}}, \tilde{\varphi})\right\|_{X_{T}}=M<\frac{R}{2} .
$$

Then, a direct calculation shows

$$
\begin{aligned}
\left\|\mathcal{L}^{-1} \circ \mathcal{F}(\mathbf{v}, \varphi)\right\|_{X_{T}} & \leq\left\|\mathcal{L}^{-1} \circ \mathcal{F}(\mathbf{v}, \varphi)-\mathcal{L}^{-1} \circ \mathcal{F}(\tilde{\mathbf{v}}, \tilde{\varphi})\right\|_{X_{T}}+\left\|\mathcal{L}^{-1} \circ \mathcal{F}(\tilde{\mathbf{v}}, \tilde{\varphi})\right\|_{X_{T}} \\
& <\left\|\mathcal{L}^{-1}\right\|_{\mathcal{L}\left(Y_{T}, X_{T}\right)}\|\mathcal{F}(\mathbf{v}, \varphi)-\mathcal{F}(\tilde{\mathbf{v}}, \tilde{\varphi})\|_{Y_{T}}+\frac{R}{2} \\
& \leq\left\|\mathcal{L}^{-1}\right\|_{\mathcal{L}\left(Y_{T}, X_{T}\right)} C(R, T)\|(\mathbf{v}, \varphi)-(\tilde{\mathbf{v}}, \tilde{\varphi})\|_{X_{T}}+\frac{R}{2}<R
\end{aligned}
$$

for every $(\mathbf{v}, \varphi) \in \overline{B_{R}^{X_{T}}(0)}$, where we used the estimate for the Lipschitz continuity of $\mathcal{F}$. This shows that $\mathcal{L}^{-1} \circ \mathcal{F}(\mathbf{v}, \varphi)$ is in $\overline{B_{R}^{X_{T}}(0)}$ for every $(\mathbf{v}, \varphi) \in \overline{B_{R}^{X_{T}}(0)}$, i.e.,

$$
\mathcal{L}^{-1} \circ \mathcal{F}: \overline{B_{R}^{X_{T}}(0)} \rightarrow \overline{B_{R}^{X_{T}}(0)} .
$$

For applying the Banach fixed-point theorem, it remains to show that the mapping $\mathcal{L}^{-1} \circ F: B_{R}^{X_{T}}(0) \rightarrow B_{R}^{X_{T}}(0)$ is a contraction. To this end, let $\left(\mathbf{v}_{i}, \varphi_{i}\right) \in B_{R}^{X_{T}}(0)$ be given for $i=1,2$. Then, it holds

$$
\begin{aligned}
& \left\|\mathcal{L}^{-1} \circ \mathcal{F}\left(\mathbf{v}_{1}, \varphi_{1}\right)-\mathcal{L}^{-1} \circ \mathcal{F}\left(\mathbf{v}_{2}, \varphi_{2}\right)\right\|_{X_{T}} \\
& \quad \leq\left\|\mathcal{L}^{-1}\right\|_{\mathcal{L}\left(Y_{T}, X_{T}\right)} C(R, T)\left\|\left(\mathbf{v}_{1}, \varphi_{1}\right)-\left(\mathbf{v}_{2}, \varphi_{2}\right)\right\|_{X_{T}} \\
& \quad<\frac{1}{2}\left\|\left(\mathbf{v}_{1}, \varphi_{1}\right)-\left(\mathbf{v}_{2}, \varphi_{2}\right)\right\|_{X_{T}},
\end{aligned}
$$


which shows the statement. Hence, the Banach fixed-point theorem can be applied and yields some $(\mathbf{v}, \varphi) \in \overline{B_{R}^{X_{T}}(0)} \subseteq X_{T}$ such that the fixed-point equation (10) holds, which implies that $(\mathbf{v}, \varphi)$ is a strong solution for Eqs. (1)-(4).

Finally, in order to show uniqueness in $X_{T}$, let $(\hat{\mathbf{v}}, \hat{\varphi}) \in X_{T}$ be another solution. Choose $\hat{R} \geq R$ such that $(\hat{\mathbf{v}}, \hat{\varphi}) \in \overline{B_{\hat{R}}^{X_{T}}(0)}$. Then by the previous arguments, we can find some $\hat{T} \in(0, T]$ such that (10) has a unique solution. This implies $\left.(\hat{\mathbf{v}}, \hat{\varphi})\right|_{[0, \hat{T}]}=$ $\left.(\mathbf{v}, \varphi)\right|_{[0, \hat{T}]}$. A standard continuation argument shows that the solutions coincide for all $t \in[0, T]$.

\section{Lipschitz continuity of $\mathcal{F}$}

Before we continue, we study in which Banach spaces $\mathbf{v}, \varphi, \nabla \varphi, m(\varphi)$ and so on are bounded.

Note that in the definition of $X_{T}^{2}, p$ has to be larger than 4 because we will need to estimate terms like $\nabla \Delta \varphi \cdot \nabla \mathbf{v}$, where $p=2$ is not sufficient for the analysis and therefore we need to choose $p>2$. But for most terms in the analysis $p=2$ would be sufficient and $4<p<6$ would not be necessary. Nevertheless, for consistency all calculations are done for the case $4<p<6$.

Due to (8), it holds

$$
\mathbf{v} \in X_{T}^{1} \hookrightarrow B U C\left([0, T] ; B_{22}^{1}(\Omega)\right)=B U C\left([0, T] ; H^{1}(\Omega)\right),
$$

where we used $B_{22}^{s}(\Omega)=H_{2}^{s}(\Omega)$ for every $s \in \mathbb{R}$. In particular, this implies

$$
\begin{aligned}
& \nabla \mathbf{v} \in L^{\infty}\left(0, T ; L^{2}(\Omega)\right) \cap L^{2}\left(0, T ; L^{6}(\Omega)\right) \hookrightarrow L^{\frac{8}{3}}\left(0, T ; L^{4}(\Omega)\right), \\
& \nabla \mathbf{v} \in L^{\infty}\left(0, T ; L^{2}(\Omega)\right) \cap L^{2}\left(0, T ; L^{6}(\Omega)\right) \hookrightarrow L^{4}\left(0, T ; L^{3}(\Omega)\right) .
\end{aligned}
$$

Let $\varphi \in X_{T}^{2}$ be given. From it (8) follows

$$
\varphi \in L^{p}\left(0, T ; W_{p, N}^{4}(\Omega)\right) \cap W_{p}^{1}\left(0, T ; L^{p}(\Omega)\right) \hookrightarrow B U C\left([0, T] ; W_{p}^{4-\frac{4}{p}}(\Omega)\right) .
$$

This implies

$$
\nabla \Delta \varphi \in B U C\left([0, T] ; W_{p}^{1-\frac{4}{p}}(\Omega)\right)
$$

since $p>4$. Note that when we write " $\varphi$ is bounded in $Z$ " for some function space $Z$, we mean that the set of all functions $\left\{\varphi \in X_{T}^{2}:\|\varphi\|_{X_{T}^{2}} \leq R\right\}$ is bounded in $Z$ in such a way that the upper bound only depends on $R$ and not on $T$, i.e., there exists $C(R)>0$ such that $\|\varphi\|_{Z} \leq C(R)$ for every $\varphi \in X_{T}^{2}$ with $\|\varphi\|_{X_{T}^{2}} \leq R$.

First of all, we have

$$
\varphi \in W_{p}^{1}\left(0, T ; L^{p}(\Omega)\right) \hookrightarrow C^{0,1-\frac{1}{p}}\left([0, T] ; L^{p}(\Omega)\right) .
$$


Moreover, we already know that $\varphi \in B U C\left([0, T] ; W_{p}^{4-\frac{4}{p}}(\Omega)\right)$ and we have

$$
\left(B_{p p}^{4-\frac{4}{p}}(\Omega), L^{p}(\Omega)\right)_{\theta, 2}=B_{p 2}^{3}(\Omega) \hookrightarrow W_{p}^{3}(\Omega)
$$

together with the estimate

$$
\|\varphi(t)\|_{W_{p}^{3}(\Omega)} \leq C\|\varphi(t)\|_{W_{p}^{4-\frac{4}{p}}(\Omega)}^{1-\theta}\|\varphi(t)\|_{L^{p}(\Omega)}^{\theta}
$$

for every $t \in[0, T]$. Hence, Lemma 1 implies

$$
\begin{aligned}
& \varphi \in C^{0,1-\frac{1}{p}}\left([0, T] ; L^{p}(\Omega)\right) \cap C\left([0, T] ; W_{p}^{4-\frac{4}{p}}(\Omega)\right) \\
& \hookrightarrow C^{0,\left(1-\frac{1}{p}\right) \theta}\left([0, T] ; W_{p}^{3}(\Omega)\right) .
\end{aligned}
$$

Because of $W_{p}^{3}(\Omega) \hookrightarrow C^{2}(\bar{\Omega})$ for $d=2,3$ due to $4<p<6$, we obtain that

$$
\varphi \text { is bounded in } C\left([0, T] ; C^{2}(\bar{\Omega})\right) \text {. }
$$

In the nonlinear operator $\mathcal{F}: X_{T} \rightarrow Y_{T}$, the terms $\eta(\varphi), \eta\left(\varphi_{0}\right), m(\varphi), m\left(\varphi_{0}\right)$ and $W^{\prime}(\varphi)$ appear. Hence, we need to know in which spaces these terms are bounded in the sense that there is a constant $C(R)>0$, which does not depend on $T$, such that the norms of these terms in a certain Banach space are bounded by $C(R)$ for every $(\mathbf{v}, \varphi) \in X_{T}$ with $\|(\mathbf{v}, \varphi)\|_{X_{T}} \leq R$.

Due to (17) and because the embedding constant only depends on $R$, it holds

$$
\|\varphi(t)\|_{W_{p}^{3}(\Omega)} \leq C(R)
$$

for every $t \in[0, T]$ and $\varphi \in X_{T}^{2}$ with $\|\varphi\|_{X_{T}^{2}} \leq R$. Hence, Theorem 3 yields

$$
\|f(\varphi(t))\|_{W_{p}^{3}(\Omega)},\left\|f\left(\varphi_{0}\right)\right\|_{W_{p}^{3}(\Omega)},\left\|W^{\prime}(\varphi(t))\right\|_{W_{p}^{3}(\Omega)} \leq C(R)
$$

for every $t \in[0, T]$ and every $\varphi \in X_{T}^{2}$ with $\|\varphi\|_{X_{T}^{2}} \leq R$, where $f \in\{\eta, m\}$. Thus,

$$
f(\varphi), f\left(\varphi_{0}\right), f^{\prime}(\varphi), W^{\prime}(\varphi) \text { are bounded in } L^{\infty}\left(0, T ; W_{p}^{3}(\Omega)\right)
$$

for $f \in\{\eta, m\}$. Moreover, Theorem 3 yields the existence of $L>0$ such that

$$
\left\|f\left(\varphi_{1}(t)\right)-f\left(\varphi_{2}(t)\right)\right\|_{W_{p}^{3}(\Omega)} \leq L\left\|\varphi_{1}(t)-\varphi_{2}(t)\right\|_{W_{p}^{3}(\Omega)}
$$

for every $t \in[0, T], \varphi_{1}, \varphi_{2} \in X_{T}^{2}$ and $f \in\left\{\eta, m, W^{\prime}\right\}$.

In the next step, we want to show that $f(\varphi)$ is bounded in $X_{T}^{2}$ and therefore, the same embeddings hold as for $\varphi$, where $f \in\left\{\eta, m, W^{\prime}\right\}$. Note that from now on until the end of the proof of the interpolation result for $f(\varphi)$, we always use some general $f \in C_{b}^{4}(\mathbb{R})$. But all these embeddings are valid for $f \in\left\{\eta, m, W^{\prime}\right\}$. We want to prove 
that if it holds $\varphi \in X_{T}^{2}$ with $\|\varphi\|_{X_{T}^{2}} \leq R$, then there exists a constant $C(R)>0$ such that $\|f(\varphi)\|_{X_{T}^{2}} \leq C(R)$. To this end, let $\varphi \in X_{T}^{2}$ be given with $\|\varphi\|_{X_{T}^{2}} \leq R$. Since we already know $\varphi \in C\left([0, T] ; C^{2}(\bar{\Omega})\right)$, cf. (18), we can conclude

$$
\|\varphi(t)\|_{C^{2}(\bar{\Omega})} \leq C(R)
$$

for all $t \in[0, T]$. Hence, it holds $f(\varphi(t)) \in C^{2}(\bar{\Omega})$ for every $t \in[0, T]$ and

$$
\nabla f\left((\varphi(t))=f^{\prime}(\varphi(t)) \nabla \varphi(t)\right.
$$

Due to (19), $f^{\prime}(\varphi)$ is bounded in $L^{\infty}\left(0, T ; W_{p}^{3}(\Omega)\right)$. In particular, this implies $\left\|f^{\prime}(\varphi(t))\right\|_{W_{p}^{3}(\Omega)} \leq C(R)$ for a.e. $t \in(0, T)$ and a constant $C(R)>0$. Since it holds $\varphi \in L^{p}\left(0, T ; W_{p}^{4}(\Omega)\right)$, it follows $\nabla \varphi(t) \in W_{p}^{3}(\Omega)$ for a.e. $t \in(0, T)$. Since $W_{p}^{3}(\Omega)$ is a Banach algebra, we obtain $f^{\prime}(\varphi(t)) \nabla \varphi(t) \in W_{p}^{3}(\Omega)$ for a.e. $t \in(0, T)$ together with the estimate

$$
\|\nabla f(\varphi(t))\|_{W_{p}^{3}(\Omega)}=\left\|f^{\prime}(\varphi(t)) \nabla \varphi(t)\right\|_{W_{p}^{3}(\Omega)} \leq C\left\|f^{\prime}(\varphi(t))\right\|_{W_{p}^{3}(\Omega)}\|\nabla \varphi(t)\|_{W_{p}^{3}(\Omega)}
$$

for a.e. $t \in(0, T)$ and every $\varphi \in X_{T}^{2}$ with $\|\varphi\|_{X_{T}^{2}} \leq R$. Since $f^{\prime}(\varphi)$ is bounded in $L^{\infty}\left(0, T ; W_{p}^{3}(\Omega)\right)$ and $\nabla \varphi$ is bounded in $L^{p}\left(0, T ; W_{p}^{3}(\Omega)\right)$, the estimate above implies the boundedness of $\nabla f(\varphi)$ in $L^{p}\left(0, T ; W_{p}^{3}(\Omega)\right)$, i.e., there exists $C(R)>0$ such that

$$
\|\nabla f(\varphi)\|_{L^{p}\left(0, T ; W_{p}^{3}(\Omega)\right)} \leq C(R) \quad \text { for all } \varphi \in X_{T}^{2} \text { with }\|\varphi\|_{X_{T}^{2}} \leq R .
$$

Altogether this implies that

$$
f(\varphi) \text { is bounded in } L^{p}\left(0, T ; W_{p}^{4}(\Omega)\right) .
$$

Analogously, we can conclude from the boundedness of $\varphi$ in $W_{p}^{1}\left(0, T ; L^{p}(\Omega)\right)$ that $f(\varphi)$ is also bounded in $W_{p}^{1}\left(0, T ; L^{p}(\Omega)\right)$ because of $\frac{\mathrm{d}}{\mathrm{d} t} f(\varphi(t))=f^{\prime}(\varphi(t)) \partial_{t} \varphi(t)$, where $f^{\prime}(\varphi)$ is bounded in $C^{0}\left(\bar{Q}_{T}\right)$. Thus, the same interpolation result holds as in (17), i.e.,

$$
f(\varphi) \text { is bounded in } C^{0,\left(1-\frac{1}{p}\right) \theta}\left([0, T] ; W_{p}^{3}(\Omega)\right)
$$

where $\theta:=\frac{\frac{4}{p}-1}{\frac{4}{p}-4}$.

Proof of Proposition 1. Let $\left(\mathbf{v}_{i}, \varphi_{i}\right) \in X_{T}$ with $\left\|\left(\mathbf{v}_{i}, \varphi_{i}\right)\right\|_{X_{T}} \leq R, i=1,2$, be given. Then, it holds

$$
\begin{aligned}
& \left\|\mathcal{F}\left(\mathbf{v}_{1}, \varphi_{1}\right)-\mathcal{F}\left(\mathbf{v}_{2}, \varphi_{2}\right)\right\|_{Y_{T}}=\left\|\mathbb{P}_{\sigma}\left(F_{1}\left(\mathbf{v}_{1}, \varphi_{1}\right)-F_{1}\left(\mathbf{v}_{2}, \varphi_{2}\right)\right)\right\|_{L^{2}\left(Q_{T}\right)} \\
& +\|\left(\nabla \varphi_{2} \cdot \mathbf{v}_{2}-\nabla \varphi_{1} \cdot \mathbf{v}_{1}\right)+\frac{1}{\varepsilon} \operatorname{div}\left(m\left(\varphi_{1}\right) \nabla W^{\prime}\left(\varphi_{1}\right)-m\left(\varphi_{2}\right) \nabla W^{\prime}\left(\varphi_{2}\right)\right) \\
& +\varepsilon m\left(\varphi_{0}\right) \Delta^{2}\left(\varphi_{1}-\varphi_{2}\right)+\varepsilon \operatorname{div}\left(m\left(\varphi_{2}\right) \nabla \Delta \varphi_{2}-m\left(\varphi_{1}\right) \nabla \Delta \varphi_{2}\right) \|_{L^{p}\left(Q_{T}\right)}
\end{aligned}
$$


For the sake of clarity, we study both summands in (22) separately and begin with the first one. Recall that the operator $F_{1}$ is defined by

$$
\begin{aligned}
F_{1}(\mathbf{v}, \varphi)= & \rho_{0} \partial_{t} \mathbf{v}-\rho \partial_{t} \mathbf{v}-\operatorname{div}\left(2 \eta\left(\varphi_{0}\right) D \mathbf{v}\right)+\operatorname{div}(2 \eta(\varphi) D \mathbf{v})-\varepsilon \Delta \varphi \nabla \varphi \\
& -\left(\left(\rho \mathbf{v}+\frac{\tilde{\rho}_{1}-\tilde{\rho}_{2}}{2} m(\varphi) \nabla\left(\frac{1}{\varepsilon} W^{\prime}(\varphi)-\varepsilon \Delta \varphi\right)\right) \cdot \nabla\right) \mathbf{v}
\end{aligned}
$$

and that it holds $\left\|\mathbb{P}_{\sigma}\right\|_{\mathcal{L}\left(L^{2}(\Omega)^{d}, L_{\sigma}^{2}(\Omega)\right)} \leq 1$ for the Helmholtz projection $\mathbb{P}_{\sigma}$. We estimate $\left\|\mathbb{P}_{\sigma}\left(F_{1}\left(\mathbf{v}_{1}, \varphi_{1}\right)-F_{1}\left(\mathbf{v}_{2}, \varphi_{2}\right)\right)\right\|_{L^{2}\left(Q_{T}\right)}$ :

For the first two terms, we can calculate

$$
\begin{aligned}
& \left\|\rho_{0} \partial_{t} \mathbf{v}_{1}-\rho\left(\varphi_{1}\right) \partial_{t} \mathbf{v}_{1}-\rho_{0} \partial_{t} \mathbf{v}_{2}+\rho\left(\varphi_{2}\right) \partial_{t} \mathbf{v}_{2}\right\|_{L^{2}\left(Q_{T}\right)} \\
& \quad \leq\left\|\left(\rho_{0}-\rho\left(\varphi_{1}\right)\right) \partial_{t}\left(\mathbf{v}_{1}-\mathbf{v}_{2}\right)\right\|_{L^{2}\left(Q_{T}\right)}+\left\|\left(\rho\left(\varphi_{1}\right)-\rho\left(\varphi_{2}\right)\right) \partial_{t} \mathbf{v}_{2}\right\|_{L^{2}\left(Q_{T}\right)} .
\end{aligned}
$$

Since it holds $\partial_{t} \mathbf{v}_{i} \in L^{2}\left(0, T ; L_{\sigma}^{2}(\Omega)\right), i=1,2$, we need to estimate every $\rho$-term in the $L^{\infty}$-norm. To this end, we use that $\rho$ is affine linear and

$$
\varphi_{i} \text { is bounded in } C^{0,\left(1-\frac{1}{p}\right) \theta}\left([0, T] ; W_{p}^{3}(\Omega)\right) \hookrightarrow C^{0,\left(1-\frac{1}{p}\right) \theta}\left([0, T] ; C^{2}(\bar{\Omega})\right)
$$

for $i=1,2$ and $\theta=\frac{\frac{4}{p}-1}{\frac{4}{p}-4}$, cf. (17). Then, we obtain for the first summand

$$
\begin{aligned}
\left\|\left(\rho_{0}-\rho\left(\varphi_{1}\right)\right) \partial_{t}\left(\mathbf{v}_{1}-\mathbf{v}_{2}\right)\right\|_{L^{2}\left(Q_{T}\right)} & \leq\left\|\rho\left(\varphi_{0}\right)-\rho\left(\varphi_{1}\right)\right\|_{L^{\infty}\left(Q_{T}\right)}\left\|\partial_{t}\left(\mathbf{v}_{1}-\mathbf{v}_{2}\right)\right\|_{L^{2}\left(Q_{T}\right)} \\
& \leq C \sup _{t \in[0, T]}\left\|\varphi_{1}(0)-\varphi_{1}(t)\right\|_{L^{\infty}(\Omega)}\left\|\mathbf{v}_{1}-\mathbf{v}_{2}\right\|_{X_{T}^{1}} \\
& \leq C T^{\left(1-\frac{1}{p}\right) \theta}\left\|\varphi_{1}\right\|_{C^{0,\left(1-\frac{1}{p}\right) \theta}\left([0, T] ; C^{2}(\bar{\Omega})\right)}\left\|\mathbf{v}_{1}-\mathbf{v}_{2}\right\|_{X_{T}^{1}} \\
& \leq C R T^{\left(1-\frac{1}{p}\right) \theta}\left\|\mathbf{v}_{1}-\mathbf{v}_{2}\right\|_{X_{T}^{1}} .
\end{aligned}
$$

Analogously, the second term can be estimated by

$$
\begin{aligned}
& \left\|\left(\rho\left(\varphi_{1}\right)-\rho\left(\varphi_{2}\right)\right) \partial_{t} \mathbf{v}_{2}\right\|_{L^{2}\left(Q_{T}\right)} \leq\left\|\rho\left(\varphi_{1}\right)-\rho\left(\varphi_{2}\right)\right\|_{L^{\infty}\left(Q_{T}\right)}\left\|\mathbf{v}_{2}\right\|_{X_{T}^{1}} \\
& \quad \leq C \sup _{t \in[0, T]}\left\|\left(\varphi_{1}(t)-\varphi_{2}(t)\right)-\left(\varphi_{1}(0)-\varphi_{2}(0)\right)\right\|_{L^{\infty}(\Omega)}\left\|\mathbf{v}_{2}\right\|_{X_{T}^{1}} \\
& \quad \leq C R T^{\left(1-\frac{1}{p}\right) \theta}\left\|\varphi_{1}-\varphi_{2}\right\|_{C^{0,\left(1-\frac{1}{p}\right) \theta}\left([0, T] ; C^{2}(\bar{\Omega})\right)} \\
& \quad \leq C R T^{\left(1-\frac{1}{p}\right) \theta}\left\|\varphi_{1}-\varphi_{2}\right\|_{X_{T}^{2}} .
\end{aligned}
$$

Here, we used the fact that $\varphi_{1}(0)=\varphi_{0}=\varphi_{2}(0)$ for $\varphi_{i} \in X_{T}^{2}, i=1,2$.

The next term of $\left\|\mathbb{P}_{\sigma}\left(F_{1}\left(\mathbf{v}_{1}, \varphi_{1}\right)-F_{1}\left(\mathbf{v}_{2}, \varphi_{2}\right)\right)\right\|_{L^{2}\left(Q_{T}\right)}$ is given by

$$
\begin{aligned}
& \left\|\left(\operatorname{div}\left(2 \eta\left(\varphi_{0}\right) D \mathbf{v}_{2}\right)-\operatorname{div}\left(2 \eta\left(\varphi_{0}\right) D \mathbf{v}_{1}\right)\right)+\left(\operatorname{div}\left(2 \eta\left(\varphi_{1}\right) D \mathbf{v}_{1}\right)-\operatorname{div}\left(2 \eta\left(\varphi_{2}\right) D \mathbf{v}_{2}\right)\right)\right\|_{Y_{T}^{1}} \\
& \quad \leq\left\|\operatorname{div}\left(2\left(\eta\left(\varphi_{0}\right)-\eta\left(\varphi_{1}\right)\right)\left(D \mathbf{v}_{2}-D \mathbf{v}_{1}\right)\right)\right\|_{Y_{T}^{1}}+\left\|\operatorname{div}\left(2\left(\left(\eta\left(\varphi_{1}\right)-\eta\left(\varphi_{2}\right)\right) D \mathbf{v}_{2}\right)\right)\right\|_{Y_{T}^{1}}
\end{aligned}
$$


In the next step, we apply the divergence on the $\eta\left(\varphi_{i}\right)$ - and $D \mathbf{v}_{i}$-terms, and for the sake of clarity, we study both terms in the previous inequality separately. For the first one, we use $\eta(\varphi) \in C^{0,\left(1-\frac{1}{p}\right) \theta}\left([0, T] ; W_{p}^{3}(\Omega)\right)$ with $\theta=\frac{\frac{4}{p}-1}{\frac{4}{p}-4}$, cf. (21), to obtain

$$
\begin{aligned}
& \left\|\operatorname{div}\left(2\left(\eta\left(\varphi_{0}\right)-\eta\left(\varphi_{1}\right)\right)\left(D \mathbf{v}_{2}-D \mathbf{v}_{1}\right)\right)\right\|_{Y_{T}^{1}} \\
& \leq\left\|2 \nabla\left(\eta\left(\varphi_{0}\right)-\eta\left(\varphi_{1}\right)\right) \cdot\left(D \mathbf{v}_{2}-D \mathbf{v}_{1}\right)\right\|_{Y_{T}^{1}}+\left\|\left(\eta\left(\varphi_{0}\right)-\eta\left(\varphi_{1}\right)\right) \Delta\left(\mathbf{v}_{2}-\mathbf{v}_{1}\right)\right\|_{Y_{T}^{1}} \\
& \leq C \sup _{t \in[0, T]}\left\|\nabla \eta\left(\varphi_{1}(0)\right)-\nabla \eta\left(\varphi_{1}(t)\right)\right\|_{C^{1}(\bar{\Omega})}\left\|D \mathbf{v}_{2}-D \mathbf{v}_{1}\right\|_{L^{2}\left(0, T ; H^{1}(\Omega)\right)} \\
& +C \sup _{t \in(0, T)}\left\|\eta\left(\varphi_{1}(0)\right)-\eta\left(\varphi_{1}(t)\right)\right\|_{C^{2}(\bar{\Omega})}\left\|\Delta\left(\mathbf{v}_{2}-\mathbf{v}_{1}\right)\right\|_{L^{2}\left(0, T ; L^{2}(\Omega)\right)}
\end{aligned}
$$

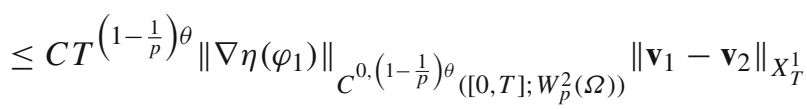

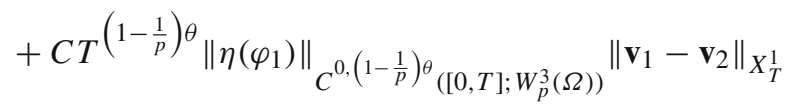

$$
\begin{aligned}
& \leq C R\left(T^{\left(1-\frac{1}{p}\right) \theta}+T^{\left(1-\frac{1}{p}\right) \theta}\right)\left\|\mathbf{v}_{1}-\mathbf{v}_{2}\right\|_{X_{T}^{1}} \text {. }
\end{aligned}
$$

Analogously, as before we can estimate the second summand by

$$
\begin{aligned}
& \left\|\operatorname{div}\left(2\left(\left(\eta\left(\varphi_{1}\right)-\eta\left(\varphi_{2}\right)\right) D \mathbf{v}_{2}\right)\right)\right\|_{Y_{T}^{1}} \\
& \quad \leq 2\left\|\eta^{\prime}\left(\varphi_{1}\right)\left(\nabla \varphi_{1}-\nabla \varphi_{2}\right) \cdot D \mathbf{v}_{2}\right\|_{Y_{T}^{1}}+2\left\|\left(\eta^{\prime}\left(\varphi_{1}\right)-\eta^{\prime}\left(\varphi_{2}\right)\right) \nabla \varphi_{2} \cdot D \mathbf{v}_{2}\right\|_{Y_{T}^{1}} \\
& \quad+\left\|\left(\eta\left(\varphi_{1}\right)-\eta\left(\varphi_{2}\right)\right) \Delta \mathbf{v}_{2}\right\|_{Y_{T}^{1}} .
\end{aligned}
$$

For the sake of clarity, we study these three terms separately again. Firstly,

$$
\begin{aligned}
& \left\|\eta^{\prime}\left(\varphi_{1}\right)\left(\nabla \varphi_{1}-\nabla \varphi_{2}\right) \cdot D \mathbf{v}_{2}\right\|_{Y_{T}^{1}} \leq C(R)\|\| D \mathbf{v}_{2}\left\|_{L^{2}(\Omega)}\right\| \nabla \varphi_{1}-\nabla \varphi_{2}\left\|_{C^{1}(\bar{\Omega})}\right\|_{L^{2}(0, T)} \\
& \quad \leq C(R) \sup _{t \in[0, T]}\left\|\nabla\left(\varphi_{1}(t)-\varphi_{2}(t)\right)-\nabla\left(\varphi_{1}(0)-\varphi_{2}(0)\right)\right\|_{C^{1}(\bar{\Omega})}\left\|D \mathbf{v}_{2}\right\|_{L^{2}\left(0, T ; L^{2}(\Omega)\right)} \\
& \quad \leq C(R) T^{\left(1-\frac{1}{p}\right) \theta}\left\|\nabla \varphi_{1}-\nabla \varphi_{2}\right\|_{C}^{\left(1-\frac{1}{p}\right) \theta}\left\|_{\left([0, T] ; W_{p}^{2}(\Omega)\right)}\right\| \mathbf{v}_{2} \|_{X_{T}^{1}} \\
& \quad \leq C(R) T^{\left(1-\frac{1}{p}\right) \theta}\left\|\varphi_{1}-\varphi_{2}\right\|_{X_{T}^{2}},
\end{aligned}
$$

where we used in the first step that $\eta^{\prime}(\varphi)$ is bounded in $C\left([0, T] ; C^{2}(\bar{\Omega})\right)$. Furthermore, (20) together with

$$
\varphi \in C^{0,\left(1-\frac{1}{p}\right) \theta}\left([0, T] ; W_{p}^{3}(\Omega)\right) \hookrightarrow C\left([0, T] ; C^{2}(\bar{\Omega})\right)
$$


implies

$$
\begin{aligned}
& \left\|\left(\eta^{\prime}\left(\varphi_{1}\right)-\eta^{\prime}\left(\varphi_{2}\right)\right) \nabla \varphi_{2} \cdot D \mathbf{v}_{2}\right\|_{Y_{T}^{1}} \\
& \quad \leq \sup _{t \in[0, T]}\left\|\eta^{\prime}\left(\varphi_{1}\right)-\eta^{\prime}\left(\varphi_{2}\right)\right\|_{W_{p}^{3}(\Omega)}\left\|\nabla \varphi_{2}\right\|_{C\left([0, T] ; C^{1}(\bar{\Omega})\right)}\left\|D \mathbf{v}_{2}\right\|_{L^{2}\left(Q_{T}\right)} \\
& \quad \leq C(R) \sup _{t \in[0, T]}\left\|\varphi_{1}(t)-\varphi_{2}(t)\right\|_{W_{p}^{3}(\Omega)} \leq C(R) T^{\left(1-\frac{1}{p}\right) \theta}\left\|\varphi_{1}-\varphi_{2}\right\|_{X_{T}^{2}}
\end{aligned}
$$

since $\varphi_{1}(0)-\varphi_{2}(0)=0$. Analogously, to the second summand we can estimate the third one by

$$
\left\|\left(\eta\left(\varphi_{1}\right)-\eta\left(\varphi_{2}\right)\right) \Delta \mathbf{v}_{2}\right\|_{Y_{T}} \leq C(R) T^{\left(1-\frac{1}{p}\right) \theta}\left\|\varphi_{1}-\varphi_{2}\right\|_{X_{T}^{2}},
$$

which shows the statement for the second term.

For the third term, we obtain

$$
\begin{aligned}
& \left\|\rho\left(\varphi_{2}\right) \mathbf{v}_{2} \cdot \nabla \mathbf{v}_{2}-\rho\left(\varphi_{1}\right) \mathbf{v}_{1} \cdot \nabla \mathbf{v}_{1}\right\|_{Y_{T}^{1}} \\
& \quad \leq\left\|\left(\rho\left(\varphi_{2}\right)-\rho\left(\varphi_{1}\right)\right) \mathbf{v}_{2} \cdot \nabla \mathbf{v}_{2}\right\|_{Y_{T}^{1}}+\left\|\rho\left(\varphi_{1}\right)\left(\mathbf{v}_{2}-\mathbf{v}_{1}\right) \cdot \nabla \mathbf{v}_{2}\right\|_{Y_{T}^{1}} \\
& \left.\quad+\| \rho\left(\varphi_{1}\right) \mathbf{v}_{1} \cdot\left(\nabla \mathbf{v}_{2}-\nabla \mathbf{v}_{1}\right)\right) \|_{Y_{T}^{1}} .
\end{aligned}
$$

We estimate these three terms separately again. For the first term, we use that $\mathbf{v}_{2}$ is bounded in $L^{\infty}\left(0, T ; L^{6}(\Omega)\right)$, cf. (12), and $\nabla \mathbf{v}_{2}$ is bounded in $L^{2}\left(0, T ; L^{6}(\Omega)\right)$ together with (20). Thus,

$$
\begin{aligned}
& \left\|\left(\rho\left(\varphi_{2}\right)-\rho\left(\varphi_{1}\right)\right) \mathbf{v}_{2} \cdot \nabla \mathbf{v}_{2}\right\|_{Y_{T}^{1}} \\
& \quad \leq C(R) T^{\left(1-\frac{1}{p}\right) \theta}\left\|\varphi_{2}-\varphi_{1}\right\|_{C^{0,\left(1-\frac{1}{p}\right) \theta}\left([0, T] ; W_{p}^{3}(\Omega)\right)}\left\|\mathbf{v}_{2}\right\|_{L^{\infty}\left(0, T ; L^{6}(\Omega)\right)}\left\|\nabla \mathbf{v}_{2}\right\|_{L^{2}\left(0, T ; L^{6}(\Omega)\right)} \\
& \quad \leq C(R) T^{\left(1-\frac{1}{p}\right) \theta}\left\|\varphi_{2}-\varphi_{1}\right\|_{X_{T}^{1}} .
\end{aligned}
$$

For the second term, we use $\rho\left(\varphi_{1}\right) \in C\left([0, T] ; C^{2}(\bar{\Omega})\right), \mathbf{v}_{i} \in L^{\infty}\left(0, T ; L^{6}(\Omega)\right)$ and $\nabla \mathbf{v}_{2} \in L^{4}\left(0, T ; L^{3}(\Omega)\right)$, cf. (12) and (14), $i=1,2$. Hence,

$$
\begin{aligned}
\left\|\rho\left(\varphi_{1}\right)\left(\mathbf{v}_{2}-\mathbf{v}_{1}\right) \cdot \nabla \mathbf{v}_{2}\right\|_{Y_{T}^{1}} & \leq C(R) T^{\frac{1}{4}}\left\|\mathbf{v}_{1}-\mathbf{v}_{2}\right\|_{L^{\infty}\left(0, T ; L^{6}(\Omega)\right)}\left\|\nabla \mathbf{v}_{2}\right\|_{L^{4}\left(0, T ; L^{3}(\Omega)\right)} \\
& \leq C(R) T^{\frac{1}{4}}\left\|\mathbf{v}_{1}-\mathbf{v}_{2}\right\|_{X_{T}^{1}} .
\end{aligned}
$$

For the third term, we use the same function spaces. This implies

$$
\begin{aligned}
\left.\| \rho\left(\varphi_{1}\right) \mathbf{v}_{1} \cdot\left(\nabla \mathbf{v}_{2}-\nabla \mathbf{v}_{1}\right)\right) \|_{Y_{T}} & \leq C(R) T^{\frac{1}{4}}\left\|\nabla \mathbf{v}_{1}-\nabla \mathbf{v}_{2}\right\|_{L^{4}\left(0, T ; L^{3}(\Omega)\right)} \\
& \leq C(R) T^{\frac{1}{4}}\left\|\mathbf{v}_{1}-\mathbf{v}_{2}\right\|_{X_{T}^{1}} .
\end{aligned}
$$

Since $\frac{\tilde{\rho}_{1}-\tilde{\rho}_{2}}{2}$ is a constant, we obtain

$$
\left\|\frac{\tilde{\rho}_{1}-\tilde{\rho}_{2}}{2} m\left(\varphi_{1}\right) \nabla\left(\Delta \varphi_{1}\right) \cdot \nabla \mathbf{v}_{1}-\frac{\tilde{\rho}_{1}-\tilde{\rho}_{2}}{2} m\left(\varphi_{2}\right) \nabla\left(\Delta \varphi_{2}\right) \cdot \nabla \mathbf{v}_{2}\right\|_{Y_{T}^{1}}
$$




$$
\begin{aligned}
\leq & C\left(\left\|m\left(\varphi_{1}\right) \nabla\left(\Delta \varphi_{1}\right) \cdot\left(\nabla \mathbf{v}_{1}-\nabla \mathbf{v}_{2}\right)\right\|_{Y_{T}^{1}}\right. \\
& +\left\|m\left(\varphi_{1}\right)\left(\nabla\left(\Delta \varphi_{1}\right)-\nabla\left(\Delta \varphi_{2}\right)\right) \cdot \nabla \mathbf{v}_{2}\right\|_{Y_{T}^{1}} \\
& \left.+\left\|\left(m\left(\varphi_{1}\right)-m\left(\varphi_{2}\right)\right) \nabla\left(\Delta \varphi_{2}\right) \cdot \nabla \mathbf{v}_{2}\right\|_{Y_{T}^{1}}\right) .
\end{aligned}
$$

For the sake of clarity, we study all three terms separately again. In the following, we use $\nabla \Delta \varphi_{i} \in L^{\infty}\left(0, T ; L^{4}(\Omega)\right)$, cf. (17), $\nabla \mathbf{v}_{i} \in L^{\frac{8}{3}}\left(0, T ; L^{4}(\Omega)\right)$, cf. (13), for $i=1,2$, and $m\left(\varphi_{1}\right) \in C\left([0, T] ; C^{2}(\bar{\Omega})\right)$. Altogether this implies

$$
\begin{aligned}
& \left\|m\left(\varphi_{1}\right) \nabla\left(\Delta \varphi_{1}\right) \cdot\left(\nabla \mathbf{v}_{1}-\nabla \mathbf{v}_{2}\right)\right\|_{Y_{T}^{1}} \\
& \quad \leq C T^{\frac{1}{8}}\left\|\nabla \Delta \varphi_{1}\right\|_{L^{\infty}\left(0, T ; L^{4}(\Omega)\right)}\left\|\nabla \mathbf{v}_{1}-\nabla \mathbf{v}_{2}\right\|_{L^{\frac{8}{3}}\left(0, T ; L^{4}(\Omega)\right)} \\
& \quad \leq C(R) T^{\frac{1}{8}}\left\|\mathbf{v}_{1}-\mathbf{v}_{2}\right\|_{X_{T}^{1}} .
\end{aligned}
$$

Analogously, the second summand yields

$$
\left\|m\left(\varphi_{1}\right)\left(\nabla\left(\Delta \varphi_{1}\right)-\nabla\left(\Delta \varphi_{2}\right)\right) \cdot \nabla \mathbf{v}_{2}\right\|_{Y_{T}^{1}} \leq C(R) T^{\frac{1}{8}}\left\|\varphi_{1}-\varphi_{2}\right\|_{X_{T}^{2}} .
$$

For the last term, we use $m\left(\varphi_{i}\right) \in C^{0,\left(1-\frac{1}{p}\right) \theta}\left([0, T] ; W_{p}^{3}(\Omega)\right) \hookrightarrow C^{0}\left([0, T] ; C^{2}(\bar{\Omega})\right)$ together with (20) and obtain

$$
\begin{aligned}
& \left\|\left(m\left(\varphi_{1}\right)-m\left(\varphi_{2}\right)\right) \nabla\left(\Delta \varphi_{2}\right) \cdot \nabla \mathbf{v}_{2}\right\|_{Y_{T}^{1}} \\
& \quad \leq C(R) T^{\frac{1}{8}}\left\|\varphi_{1}(t)-\varphi_{2}(t)\right\|_{C^{0}\left([0, T] ; C^{2}(\bar{\Omega})\right)}\left\|\nabla \Delta \varphi_{2}\right\|_{L^{\infty}\left(0, T ; L^{4}(\Omega)\right.}\left\|\nabla \mathbf{v}_{2}\right\|_{L^{\frac{8}{3}}\left(0, T ; L^{4}(\Omega)\right)} \\
& \quad \leq C(R) T^{\frac{1}{8}}\left\|\varphi_{1}-\varphi_{2}\right\|_{X_{T}^{2}} .
\end{aligned}
$$

The next term has the same structure as the one before and can be estimated as

$$
\begin{aligned}
& \left\|\frac{\tilde{\rho}_{1}-\tilde{\rho}_{2}}{2} m\left(\varphi_{1}\right) \nabla\left(W^{\prime}\left(\varphi_{1}\right)\right) \cdot \nabla \mathbf{v}_{1}-\frac{\tilde{\rho}_{1}-\tilde{\rho}_{2}}{2} m\left(\varphi_{2}\right) \nabla\left(W^{\prime}\left(\varphi_{2}\right)\right) \cdot \nabla \mathbf{v}_{2}\right\|_{Y_{T}^{1}} \\
& \quad \leq C\left(\left\|m\left(\varphi_{1}\right) \nabla W^{\prime}\left(\varphi_{1}\right) \cdot\left(\nabla \mathbf{v}_{1}-\nabla \mathbf{v}_{2}\right)\right\|_{Y_{T}^{1}}\right. \\
& \quad+\left\|m\left(\varphi_{1}\right)\left(\nabla W^{\prime}\left(\varphi_{1}\right)-\nabla W^{\prime}\left(\varphi_{2}\right)\right) \cdot \nabla \mathbf{v}_{2}\right\|_{Y_{T}^{1}} \\
& \left.\quad+\left\|\left(m\left(\varphi_{1}\right)-m\left(\varphi_{2}\right)\right) \nabla W^{\prime}\left(\varphi_{2}\right) \cdot \nabla \mathbf{v}_{2}\right\|_{Y_{T}^{1}}\right) .
\end{aligned}
$$

For $\nabla \mathbf{v}_{i}, i=1,2$, we use its boundedness in $L^{4}\left(0, T ; L^{3}(\Omega)\right)$, cf. (14). Moreover, we know $\nabla W^{\prime}(\varphi) \in C\left([0, T] ; W_{p}^{3-\frac{4}{p}}(\Omega)\right)$ and $m(\varphi) \in C\left([0, T] ; C^{2}(\bar{\Omega})\right)$ for $\varphi \in B_{R}^{X_{T}^{2}}$. Using all these bounds, we can estimate the three terms in (23) separately. For the first term, we obtain

$$
\begin{aligned}
\left\|m\left(\varphi_{1}\right) \nabla W^{\prime}\left(\varphi_{1}\right) \cdot\left(\nabla \mathbf{v}_{1}-\nabla \mathbf{v}_{2}\right)\right\|_{Y_{T}^{1}} & \leq C(R) T^{\frac{1}{4}}\left\|\nabla \mathbf{v}_{1}-\nabla \mathbf{v}_{2}\right\|_{L^{4}\left(0, T ; L^{3}(\Omega)\right)} \\
& \leq C(R) T^{\frac{1}{4}}\left\|\mathbf{v}_{1}-\mathbf{v}_{2}\right\|_{X_{T}^{1}} .
\end{aligned}
$$

For the second summand in (23), we have to estimate the difference $\nabla W^{\prime}\left(\varphi_{1}\right)-$ $\nabla W^{\prime}\left(\varphi_{2}\right)$ in an appropriate manner. To this end, we use (17), (20) and $W_{p}^{2}(\Omega) \hookrightarrow$ 
$C^{1}(\bar{\Omega})$. Moreover, we use $\nabla \mathbf{v}_{2} \in L^{4}\left(0, T ; L^{3}(\Omega)\right)$, cf. (14), and $m(\varphi) \in$ $C\left([0, T] ; C^{2}(\bar{\Omega})\right)$. Then, it follows

$$
\begin{aligned}
& \left\|m\left(\varphi_{1}\right)\left(\nabla W^{\prime}\left(\varphi_{1}\right)-\nabla W^{\prime}\left(\varphi_{2}\right)\right) \cdot \nabla \mathbf{v}_{2}\right\|_{Y_{T}^{1}} \\
& \quad \leq C(R) T^{\frac{1}{4}} \sup _{t \in[0, T]}\left\|\nabla W^{\prime}\left(\varphi_{1}(t)\right)-\nabla W^{\prime}\left(\varphi_{2}(t)\right)\right\|_{W_{p}^{2}(\Omega)} \\
& \quad \leq C(R) T^{\frac{1}{4}} \sup _{t \in[0, T]}\left\|\varphi_{1}(t)-\varphi_{2}(t)\right\|_{W_{p}^{3}(\Omega)} \\
& \quad \leq C(R) T^{\frac{1}{4}+\left(1-\frac{1}{p}\right) \theta}\left\|\varphi_{1}-\varphi_{2}\right\|_{X_{T}^{2}} .
\end{aligned}
$$

So it remains to estimate the third term of (23). As before we get

$$
\begin{aligned}
\|( & \left.m\left(\varphi_{1}\right)-m\left(\varphi_{2}\right)\right) \nabla W^{\prime}\left(\varphi_{2}\right) \cdot \nabla \mathbf{v}_{2} \|_{Y_{T}^{1}} \\
\leq & C(R) T^{\frac{1}{4}+\left(1-\frac{1}{p}\right) \theta}\left\|\varphi_{1}-\varphi_{2}\right\|_{C^{0,\left(1-\frac{1}{p}\right) \theta}\left([0, T] ; W_{p}^{3}(\Omega)\right)} \\
& \left\|\nabla W^{\prime}\left(\varphi_{2}\right)\right\|_{B U C\left([0, T] ; C^{1}(\bar{\Omega})\right)}\left\|\nabla \mathbf{v}_{2}\right\|_{L^{4}\left(0, T ; L^{3}(\Omega)\right)} \\
\leq & C(R) T^{\frac{1}{4}+\left(1-\frac{1}{p}\right) \theta}\left\|\varphi_{1}-\varphi_{2}\right\|_{X_{T}^{2}},
\end{aligned}
$$

which completes the estimate for (23).

Finally, we study the last term of $\left\|\mathbb{P}_{\sigma}\left(F_{1}\left(\mathbf{v}_{1}, \varphi_{1}\right)-F_{1}\left(\mathbf{v}_{2}, \varphi_{2}\right)\right)\right\|_{L^{2}\left(Q_{T}\right)}$. It holds

$$
\left\|\Delta \varphi_{2} \nabla \varphi_{2}-\Delta \varphi_{1} \nabla \varphi_{1}\right\|_{Y_{T}} \leq\left\|\Delta \varphi_{2}\left(\nabla \varphi_{2}-\nabla \varphi_{1}\right)\right\|_{Y_{T}}+\left\|\left(\Delta \varphi_{2}-\Delta \varphi_{1}\right) \nabla \varphi_{1}\right\|_{Y_{T}} .
$$

Using $\Delta \varphi_{i} \in C\left([0, T] ; C^{0}(\bar{\Omega})\right)$ and $\nabla \varphi_{i} \in C^{0,\left(1-\frac{1}{p}\right) \theta}\left([0, T] ; W_{p}^{2}(\Omega)\right), i=1,2$, cf. (17), the first term can be estimated by

$$
\begin{aligned}
\left\|\Delta \varphi_{2}\left(\nabla \varphi_{2}-\nabla \varphi_{1}\right)\right\|_{Y_{T}^{1}} & \leq C(R) T^{\left.\frac{1}{2}+\left(1-\frac{1}{p}\right) \theta\right)}\left\|\nabla \varphi_{1}-\nabla \varphi_{2}\right\|_{C^{0,\left(1-\frac{1}{p}\right) \theta}\left([0, T] ; W_{p}^{2}(\Omega)\right)} \\
& \leq C(R) T^{\left.\frac{1}{2}+\left(1-\frac{1}{p}\right) \theta\right)}\left\|\varphi_{1}-\varphi_{2}\right\|_{X_{T}^{2}} .
\end{aligned}
$$

Analogously, the second term can be estimated by

$$
\left\|\left(\Delta \varphi_{2}-\Delta \varphi_{1}\right) \nabla \varphi_{1}\right\|_{Y_{T}} \leq C(R) T^{\left.\frac{1}{2}+\left(1-\frac{1}{p}\right) \theta\right)}\left\|\varphi_{1}-\varphi_{2}\right\|_{X_{T}^{2}} .
$$

Hence, we obtain

$$
\left\|\mathbb{P}_{\sigma}\left(F_{1}\left(\mathbf{v}_{1}, \varphi_{1}\right)-F_{1}\left(\mathbf{v}_{2}, \varphi_{2}\right)\right)\right\|_{L^{2}\left(Q_{T}\right)} \leq C(R, T)\left\|\left(\mathbf{v}_{1}-\mathbf{v}_{2}\right),\left(\varphi_{1}-\varphi_{2}\right)\right\|_{X_{T}}
$$

for a constant $C(R, T)>0$ such that $C(R, T) \rightarrow 0$ as $T \rightarrow 0$.

Remember that we study the nonlinear operator $\mathcal{F}: X_{T} \rightarrow Y_{T}$ given by

$$
\mathcal{F}(\mathbf{v}, \varphi)=\left(\begin{array}{c}
\mathbb{P}_{\sigma} F_{1}(\mathbf{v}, \varphi) \\
-\nabla \varphi \cdot \mathbf{v}+\operatorname{div}\left(\frac{1}{\varepsilon} m(\varphi) \nabla W^{\prime}(\varphi)\right)+\varepsilon m\left(\varphi_{0}\right) \Delta^{2} \varphi-\varepsilon \operatorname{div}(m(\varphi) \nabla \Delta \varphi)
\end{array}\right)
$$


and we want to show its Lipschitz continuity such that (9) holds. We already showed its Lipschitz continuity for the first part. Now, we continue to study the second one. This part has to be estimated in $L^{p}\left(0, T ; L^{p}(\Omega)\right)$ for $4<p<6$.

For the analysis, we use the boundedness of $\nabla \varphi$ in $C\left([0, T] ; C^{1}(\bar{\Omega})\right)$ and of $\mathbf{v}$ in $L^{\infty}\left(0, T ; L^{6}(\Omega)\right)$. Then, it holds

$$
\begin{aligned}
\| & \left(\nabla \varphi_{1} \cdot \mathbf{v}_{1}-\nabla \varphi_{2} \cdot \mathbf{v}_{2}\right) \|_{L^{p}\left(Q_{T}\right)} \\
& \leq\left\|\nabla \varphi_{1} \cdot\left(\mathbf{v}_{1}-\mathbf{v}_{2}\right)\right\|_{L^{p}\left(Q_{T}\right)}+\left\|\left(\nabla \varphi_{1}-\nabla \varphi_{2}\right) \cdot \mathbf{v}_{2}\right\|_{L^{p}\left(Q_{T}\right)} \\
& \leq T^{\frac{1}{p}}\left\|\nabla \varphi_{1}\right\|_{L^{\infty}\left(Q_{T}\right)}\left\|\mathbf{v}_{1}-\mathbf{v}_{2}\right\|_{L^{\infty}\left(0, T ; L^{6}(\Omega)\right)} \\
& +T^{\frac{1}{p}}\left\|\nabla \varphi_{1}-\nabla \varphi_{2}\right\|_{L^{\infty}\left(Q_{T}\right)}\left\|\mathbf{v}_{2}\right\|_{L^{\infty}\left(0, T ; L^{6}(\Omega)\right)} \\
& \leq T^{\frac{1}{p}} R\left\|\mathbf{v}_{1}-\mathbf{v}_{2}\right\|_{X_{T}^{1}}+T^{\frac{1}{p}} R\left\|\varphi_{1}-\varphi_{2}\right\|_{X_{T}^{2}}
\end{aligned}
$$

Next, we study the term $\operatorname{div}\left(m(\varphi) \nabla W^{\prime}(\varphi)\right)$. We use the boundedness of $f(\varphi)$ in $C\left([0, T] ; C^{2}(\bar{\Omega})\right) \cap C^{0,\left(1-\frac{1}{p}\right) \theta}\left([0, T] ; W_{p}^{3}(\Omega)\right)$ for $f \in\left\{m, W^{\prime}\right\}$ and $\varphi \in X_{T}^{2}$ with $\|\varphi\|_{X_{T}^{2}} \leq R$. Then, it holds

$$
\begin{aligned}
\left\|\operatorname{div}\left(m\left(\varphi_{1}\right) \nabla W^{\prime}\left(\varphi_{1}\right)\right)-\operatorname{div}\left(m\left(\varphi_{2}\right) \nabla W^{\prime}\left(\varphi_{2}\right)\right)\right\|_{Y_{T}^{2}} \\
\left.\leq C(R) \| m\left(\varphi_{1}\right) \nabla W^{\prime}\left(\varphi_{1}\right)\right)-m\left(\varphi_{2}\right) \nabla W^{\prime}\left(\varphi_{2}\right) \|_{L^{p}\left(0, T ; W_{p}^{1}(\Omega)\right)} \\
\leq C(R) T^{\frac{1}{p}} \sup _{t \in[0, T]}\left\|m\left(\varphi_{1}(t)\right)-m\left(\varphi_{2}(t)\right)\right\|_{W_{p}^{3}(\Omega)}\left\|\nabla W^{\prime}\left(\varphi_{1}\right)\right\|_{C\left([0, T] ; C^{1}(\bar{\Omega})\right)} \\
\quad+C(R) T^{\frac{1}{p}}\left\|m\left(\varphi_{2}\right)\right\|_{C\left([0, T] ; C^{2}(\bar{\Omega})\right)} \sup _{t \in[0, T]}\left\|W^{\prime}\left(\varphi_{1}(t)\right)-W^{\prime}\left(\varphi_{2}(t)\right)\right\|_{W_{p}^{3}(\Omega)} \\
\leq C(R) T^{\frac{1}{p}}\left(\sup _{t \in[0, T]}\left\|\varphi_{1}(t)-\varphi_{2}(t)\right\|_{W_{p}^{3}(\Omega)}+\sup _{t \in[0, T]}\left\|\varphi_{1}(t)-\varphi_{2}(t)\right\|_{W_{p}^{3}(\Omega)}\right) \\
\leq C(R) T^{\frac{1}{p}} \sup _{t \in[0, T]}\left\|\left(\varphi_{1}(t)-\varphi_{2}(t)\right)-\left(\varphi_{1}(0)-\varphi_{2}(0)\right)\right\|_{W_{p}^{3}(\Omega)} \\
\leq C(R) T^{\frac{1}{p}+\left(1-\frac{1}{p}\right) \theta}\left\|\varphi_{1}-\varphi_{2}\right\|_{C^{0,\left(1-\frac{1}{p}\right) \theta}\left([0, T] ; W_{p}^{3}(\Omega)\right)} \\
\leq C(R) T^{\frac{1}{p}+\left(1-\frac{1}{p}\right) \theta}\left\|\varphi_{1}-\varphi_{2}\right\|_{X_{T}^{2} .}
\end{aligned}
$$

Here, we also used $\varphi_{1}(0)=\varphi_{2}(0)=\varphi_{0}$ for $\varphi_{1}, \varphi_{2} \in X_{T}^{2}$ and (20).

Now, there remain two terms which we need to study together for the proof of the Lipschitz continuity. Due to the boundedness of $m(\varphi)$ in $B U C\left([0, T] ; W_{p}^{3}(\Omega)\right)$ and of $\nabla \Delta \varphi$ in $L^{p}\left(0, T ; W_{p}^{1}(\Omega)\right)$, Theorem 3 yields the boundedness of $m(\varphi) \nabla \Delta \varphi$ in $L^{p}\left(0, T ; W_{p}^{1}(\Omega)\right)$. Hence, this term is well-defined in the $L^{p}\left(Q_{T}\right)$-norm. We omit the prefactor $\varepsilon$ for both terms again and estimate

$$
\begin{aligned}
& \left\|m\left(\varphi_{0}\right) \Delta^{2} \varphi_{1}-m\left(\varphi_{0}\right) \Delta^{2} \varphi_{2}+\operatorname{div}\left(m\left(\varphi_{2}\right) \nabla \Delta \varphi_{2}\right)-\operatorname{div}\left(m\left(\varphi_{1}\right) \nabla \Delta \varphi_{1}\right)\right\|_{L^{p}\left(Q_{T}\right)} \\
& \quad=\|\left(m\left(\varphi_{0}\right)-m\left(\varphi_{1}\right)\right)\left(\Delta^{2} \varphi_{1}-\Delta^{2} \varphi_{2}\right)+m\left(\varphi_{1}\right) \Delta^{2} \varphi_{1}-m\left(\varphi_{1}\right) \Delta^{2} \varphi_{2}+\nabla m\left(\varphi_{2}\right) \cdot \nabla \Delta \varphi_{2}
\end{aligned}
$$




$$
\begin{aligned}
& +m\left(\varphi_{2}\right) \Delta^{2} \varphi_{2}-\nabla m\left(\varphi_{1}\right) \cdot \nabla \Delta \varphi_{1}-m\left(\varphi_{1}\right) \Delta^{2} \varphi_{1} \|_{L^{p}\left(Q_{T}\right)} \\
\leq & \|\left(m\left(\varphi_{1}(0)-m\left(\varphi_{1}\right)\right)\left(\Delta^{2} \varphi_{1}-\Delta^{2} \varphi_{2}\right)\left\|_{L^{p}\left(Q_{T}\right)}+\right\|\left(m\left(\varphi_{2}\right)-m\left(\varphi_{1}\right)\right) \Delta^{2} \varphi_{2} \|_{L^{p}}\left(Q_{T}\right)\right. \\
& +\left\|\nabla m\left(\varphi_{2}\right) \cdot \nabla \Delta \varphi_{2}-\nabla m\left(\varphi_{1}\right) \cdot \nabla \Delta \varphi_{1}\right\|_{L^{p}\left(Q_{T}\right)}
\end{aligned}
$$

For the sake of clarity, we estimate these three terms separately again. Due to the boundedness of $m\left(\varphi_{1}\right)$ in $C^{0,\left(1-\frac{1}{p}\right) \theta}\left([0, T] ; W_{p}^{3}(\Omega)\right)$ we obtain for the first term

$$
\begin{aligned}
& \|\left(m\left(\varphi_{1}(0)-m\left(\varphi_{1}\right)\right)\left(\Delta^{2} \varphi_{1}-\Delta^{2} \varphi_{2}\right) \|_{L^{p}\left(Q_{T}\right)}\right. \\
& \quad \leq \sup _{t \in(0, T)}\left\|m\left(\varphi_{1}(0)\right)-m\left(\varphi_{1}(t)\right)\right\|_{C^{0}(\bar{\Omega})}\left\|\Delta^{2} \varphi_{1}-\Delta^{2} \varphi_{2}\right\|_{L^{p}\left(Q_{T}\right)} \\
& \quad \leq C(R) T^{\left(1-\frac{1}{p}\right) \theta}\left\|m\left(\varphi_{1}\right)\right\|_{C^{0,\left(1-\frac{1}{p}\right) \theta}\left([0, T] ; W_{p}^{3}(\Omega)\right)}\left\|\varphi_{1}-\varphi_{2}\right\|_{X_{T}^{2}} .
\end{aligned}
$$

Since $m\left(\varphi_{1}\right)$ is bounded in $C^{0,\left(1-\frac{1}{p}\right) \theta}\left([0, T] ; W_{p}^{3}(\Omega)\right)$, we can estimate the second term in (24) by

$$
\begin{aligned}
& \left\|\left(m\left(\varphi_{2}\right)-m\left(\varphi_{1}\right)\right) \Delta^{2} \varphi_{2}\right\|_{L^{p}\left(Q_{T}\right) \leq} \sup _{t \in(0, T)}\left\|m\left(\varphi_{2}(t)\right)-m\left(\varphi_{1}(t)\right)\right\|_{C^{2}(\bar{\Omega})}\left\|\Delta^{2} \varphi_{2}\right\|_{L^{p}\left(Q_{T}\right)} \\
& \quad \leq C(R) \sup _{t \in(0, T)}\left\|m\left(\varphi_{2}(t)\right)-m\left(\varphi_{1}(t)\right)\right\|_{W_{p}^{3}(\Omega)} \\
& \quad \leq C(R) \sup _{t \in(0, T)}\left\|\left(\varphi_{2}(t)-\varphi_{1}(t)\right)-\left(\varphi_{2}(0)-\varphi_{1}(0)\right)\right\|_{W_{p}^{3}(\Omega)} \\
& \quad \leq C(R) T^{\left(1-\frac{1}{p}\right) \theta}\left\|\varphi_{1}-\varphi_{2}\right\|_{C^{0,\left(1-\frac{1}{p}\right) \theta}\left([0, T] ; W_{p}^{3}(\bar{\Omega})\right)}
\end{aligned}
$$

where we used (20) again in the penultimate step. Finally, we study the last term in (24). Here, we get

$$
\begin{array}{r}
\left\|\nabla m\left(\varphi_{2}\right) \cdot \nabla \Delta \varphi_{2}-\nabla m\left(\varphi_{1}\right) \cdot \nabla \Delta \varphi_{1}\right\|_{L^{p}\left(Q_{T}\right)} \\
\leq\left\|\left(\nabla m\left(\varphi_{2}\right)-\nabla m\left(\varphi_{1}\right)\right) \cdot \nabla \Delta \varphi_{2}\right\|_{L^{p}\left(Q_{T}\right)} \\
\quad+\left\|\nabla m\left(\varphi_{1}\right) \cdot\left(\nabla \Delta \varphi_{2}-\nabla \Delta \varphi_{1}\right)\right\|_{L^{p}\left(Q_{T}\right) .}
\end{array}
$$

Since $\nabla m\left(\varphi_{1}\right)$ is bounded in $C\left([0, T] ; C^{1}(\bar{\Omega})\right)$ and $\nabla \Delta \varphi_{i}$ is bounded in $C([0, T]$; $L^{p}(\Omega)$ ) for $i=1,2$, we can estimate the second summand by

$$
\begin{aligned}
& \| \nabla m\left(\varphi_{1}\right) \cdot\left(\nabla \Delta \varphi_{2}-\nabla \Delta \varphi_{1}\right) \|_{L^{p}\left(Q_{T}\right)} \\
& \quad \leq C(R) T^{\frac{1}{p}}\left\|\nabla m\left(\varphi_{1}\right)\right\|_{C\left([0, T] ; C^{1}(\bar{\Omega})\right)}\left\|\nabla \Delta \varphi_{1}-\nabla \Delta \varphi_{2}\right\|_{C\left([0, T] ; L^{p}(\Omega)\right)} \\
& \quad \leq C(R) T^{\frac{1}{p}}\left\|\varphi_{1}-\varphi_{2}\right\|_{X_{T}^{2}} .
\end{aligned}
$$

Thus, it remains to estimate the first term of (25). Here, we get

$$
\begin{aligned}
& \left\|\left(\nabla m\left(\varphi_{2}\right)-\nabla m\left(\varphi_{1}\right)\right) \cdot \nabla \Delta \varphi_{2}\right\|_{L^{p}\left(Q_{T}\right)} \\
& \quad \leq C(R) T^{\frac{1}{p}} \sup _{t \in[0, T]}\left\|\nabla m\left(\varphi_{2}(t)\right)-\nabla m\left(\varphi_{1}(t)\right)\right\|_{C^{0}(\bar{\Omega})}\left\|\nabla \Delta \varphi_{2}\right\|_{C\left([0, T] ; L^{p}(\Omega)\right)}
\end{aligned}
$$




$$
\begin{aligned}
& \leq C(R) T^{\frac{1}{p}} \sup _{t \in[0, T]}\left\|m\left(\varphi_{2}(t)\right)-m\left(\varphi_{1}(t)\right)\right\|_{W_{p}^{3}(\Omega)}\left\|\varphi_{2}\right\|_{C\left([0, T] ; W_{p}^{3}(\Omega)\right)} \\
& \leq C(R) T^{\frac{1}{p}} \sup _{t \in[0, T]}\left\|\varphi_{1}(t)-\varphi_{2}(t)\right\|_{W_{p}^{3}(\Omega)} \\
& \leq C(R) T^{\frac{1}{p}+\left(1-\frac{1}{p}\right) \theta}\left\|\varphi_{1}-\varphi_{2}\right\|_{C^{0,\left(1-\frac{1}{p}\right) \theta}\left([0, T] ; W_{p}^{3}(\Omega)\right)} .
\end{aligned}
$$

Hence, (25) is Lipschitz continuous and therefore also the second part of $\mathcal{F}$ is Lipschitz continuous. Together with the Lipschitz continuity of the first part of $\mathcal{F}$, we have shown

$$
\left\|\mathcal{F}\left(\mathbf{v}_{1}, \varphi_{1}\right)-\mathcal{F}\left(\mathbf{v}_{2}, \varphi_{2}\right)\right\|_{Y_{T}} \leq C(T, R)\left\|\left(\mathbf{v}_{1}-\mathbf{v}_{2}, \varphi_{1}-\varphi_{2}\right)\right\|_{X_{T}}
$$

for all $\left(\mathbf{v}_{i}, \varphi_{i}\right) \in X_{T}$ with $\left\|\left(\mathbf{v}_{i}, \varphi_{i}\right)\right\|_{X_{T}} \leq R, i=1,2$, and a constant $C(T, R)>0$ such that $C(T, R) \rightarrow 0$ as $T \rightarrow 0$.

\section{Existence and continuity of $\mathcal{L}^{-1}$}

In the following, we need:

Theorem 5. Let the linear, symmetric and monotone operator $\mathcal{B}$ be given from the real vector space $E$ to its algebraic dual $E^{\prime}$, and let $E_{b}^{\prime}$ be the Hilbert space which is the dual of $E$ with the seminorm

$$
|x|_{b}=\mathcal{B} x(x)^{\frac{1}{2}}, \quad x \in E .
$$

Let $A \subseteq E \times E_{b}^{\prime}$ be a relation with domain $D=\{x \in E: A(x) \neq \emptyset\}$. Let $A$ be the subdifferential, $\partial \varphi$, of a convex lower-semi-continuous function $\varphi: E_{b} \rightarrow[0, \infty]$ with $\varphi(0)=0$. Then, for each $u_{0}$ in the $E_{b}$-closure of $\operatorname{dom}(\varphi)$ and each $f \in L^{2}\left(0, T ; E_{b}^{\prime}\right)$ there is a solution $u:[0, T] \rightarrow E$ with $\mathcal{B} u \in C\left([0, T], E_{b}^{\prime}\right)$ of

$$
\frac{\mathrm{d}}{\mathrm{d} t}(\mathcal{B} u(t))+A(u(t)) \ni f(t), \quad 0<t<T,
$$

with

$$
\varphi \circ u \in L^{1}(0, T), \sqrt{t} \frac{\mathrm{d}}{\mathrm{d} t} \mathcal{B} u(\cdot) \in L^{2}\left(0, T ; E_{b}^{\prime}\right), u(t) \in D \text {, a.e. } t \in[0, T],
$$

and $\mathcal{B} u(0)=\mathcal{B} u_{0}$. If in addition $u_{0} \in \operatorname{dom}(\varphi)$, then

$$
\varphi \circ u \in L^{\infty}(0, T), \quad \frac{\mathrm{d}}{\mathrm{d} t} \mathcal{B} u \in L^{2}\left(0, T ; E_{b}^{\prime}\right) .
$$

The proof of this theorem can be found in [15, Chapter IV, Theorem 6.1].

To prove Theorem 4, we need to show the existence of $(\tilde{\mathbf{v}}, \tilde{\varphi}) \in X_{T}$ such that (11) holds and to prove that $\mathcal{L}: X_{T} \rightarrow Y_{T}$ is invertible with uniformly bounded 
inverse, i.e., there exists a constant $C>0$ which does not depend on $T$ such that $\left\|\mathcal{L}^{-1}\right\|_{\mathcal{L}\left(Y_{T}, X_{T}\right)} \leq C$. Recall that the linear operator $\mathcal{L}: X_{T} \rightarrow Y_{T}$ is defined by

$$
\mathcal{L}(\mathbf{v}, \varphi)=\left(\begin{array}{c}
\mathbb{P}_{\sigma}\left(\rho_{0} \partial_{t} \mathbf{v}\right)-\mathbb{P}_{\sigma}\left(\operatorname{div}\left(2 \eta\left(\varphi_{0}\right) D \mathbf{v}\right)\right) \\
\partial_{t} \varphi+\varepsilon m\left(\varphi_{0}\right) \Delta^{2} \varphi
\end{array}\right) .
$$

We note that the first part only depends on $\mathbf{v}$ while the second part only depends on $\varphi$. Thus, both equations can be solved separately.

To show the existence of a unique solution $\mathbf{v}$ for every right-hand side $\mathbf{f}$ in the first equation, we use Theorem 5.

So we have to specify what $E, E_{b}^{\prime}, \varphi$ and so on are in the problem we study and show that the conditions of Theorem 5 are fulfilled. Then, Theorem 5 yields the existence of a solution. More precisely, we obtain the following lemma.

Lemma 3. Let Assumption 1 hold. Then, for every $\mathbf{v}_{0} \in H_{0}^{1}(\Omega)^{d} \cap L_{\sigma}^{2}(\Omega), f \in$ $L^{2}\left(0, T ; L_{\sigma}^{2}(\Omega)\right), \varphi_{0} \in W_{r}^{1}(\Omega), r>d \geq 2$, and every $0<T<\infty$ there exists $a$ unique solution

$$
\mathbf{v} \in W_{2}^{1}\left(0, T ; L_{\sigma}^{2}(\Omega)\right) \cap L^{\infty}\left(0, T ; H_{0}^{1}(\Omega)^{d}\right)
$$

such that

$$
\begin{aligned}
\mathbb{P}_{\sigma}\left(\rho_{0} \partial_{t} \mathbf{v}\right)-\mathbb{P}_{\sigma}\left(\operatorname{div}\left(2 \eta\left(\varphi_{0}\right) D \mathbf{v}\right)\right)=f & \text { in } Q_{T}, \\
\operatorname{div}(\mathbf{v})=0 & \text { in } Q_{T}, \\
\mathbf{v} \mid \partial \Omega & =0 \quad \text { on }(0, T) \times \partial \Omega, \\
\mathbf{v}(0) & =\mathbf{v}_{0} \quad \text { in } \Omega
\end{aligned}
$$

for a.e. $(t, x)$ in $(0, T) \times \Omega$, where $\mathbf{v}(t) \in H^{2}(\Omega)^{d}$ for a.e. $t \in(0, T)$.

Proof. Since we want to solve (26)-(29) with Theorem 5, we define

$$
\mathcal{B} u:=\mathbb{P}_{\sigma}\left(\rho_{0} u\right)
$$

for $u \in E$, where we still need to specify the real vector space $E$. But as we want to have $\frac{\mathrm{d}}{\mathrm{d} t} \mathcal{B} u \in L^{2}\left(0, T ; L_{\sigma}^{2}(\Omega)\right)$, the dual space $E_{b}^{\prime}$ has to coincide with $L_{\sigma}^{2}(\Omega)$. But this can be realized by choosing $E=L_{\sigma}^{2}(\Omega)$. Then, $E_{b}^{\prime} \cong L_{\sigma}^{2}(\Omega)$ and with the notation in Theorem 5, we get the Hilbert space $E_{b}^{\prime}$ equipped with the seminorm

$$
\begin{aligned}
|\mathbf{u}|_{b}=\mathcal{B} \mathbf{u}(\mathbf{u})^{\frac{1}{2}} & =\left(\int_{\Omega} \mathbb{P}_{\sigma}\left(\rho_{0} \mathbf{u}\right) \cdot \mathbf{u} \mathrm{d} x\right)^{\frac{1}{2}}=\left(\int_{\Omega} \rho_{0} \mathbf{u} \cdot \mathbb{P}_{\sigma} \mathbf{u} \mathrm{d} x\right)^{\frac{1}{2}} \\
& =\left(\int_{\Omega} \rho_{0}|\mathbf{u}|^{2} \mathrm{~d} x\right)^{\frac{1}{2}} \cong\|\mathbf{u}\|_{L^{2}(\Omega)} .
\end{aligned}
$$

Thus, we obtain $E_{b}^{\prime} \cong L_{\sigma}^{2}(\Omega)=E_{b}$. Moreover, we define $A: \mathcal{D}(A) \rightarrow L_{\sigma}^{2}(\Omega)^{\prime} \cong$ $L_{\sigma}^{2}(\Omega)$ by

$$
(A \mathbf{u})(\mathbf{v}):= \begin{cases}-\int_{\Omega} \mathbb{P}_{\sigma} \operatorname{div}\left(2 \eta\left(\varphi_{0}\right) D \mathbf{u}\right) \cdot \mathbf{v d} x & \text { if } \mathbf{u} \in \operatorname{dom}(A) \\ \varnothing & \text { if } \mathbf{u} \notin \operatorname{dom}(A) .\end{cases}
$$


for every $\mathbf{v} \in L_{\sigma}^{2}(\Omega)$ and $\mathcal{D}(A)=H^{2}(\Omega)^{d} \cap H_{0}^{1}(\Omega)^{d} \cap L_{\sigma}^{2}(\Omega)$. Thus, we get for the relation $\mathcal{A}$ defined by $\mathcal{A}:=\{(\mathbf{u}, \mathbf{v}): \mathbf{v}=A \mathbf{u}, \mathbf{u} \in \mathcal{D}(A)\}$ the following inclusions

$$
\mathcal{A}=\left\{\left(\mathbf{u},-\mathbb{P}_{\sigma} \operatorname{div}\left(2 \eta\left(\varphi_{0}\right) D \mathbf{u}\right): \mathbf{u} \in H^{2}(\Omega)^{d} \cap H_{0}^{1}(\Omega)^{d} \cap L_{\sigma}^{2}(\Omega)\right\} \subseteq E \times E_{b}^{\prime},\right.
$$

since the term $\mathbb{P}_{\sigma} \operatorname{div}\left(2 \eta\left(\varphi_{0}\right) D \mathbf{u}\right)$ is in $L_{\sigma}^{2}(\Omega)^{\prime} \cong L_{\sigma}^{2}(\Omega)$. Now, we define $\psi$ : $L_{\sigma}^{2}(\Omega) \rightarrow[0,+\infty]$ by

$$
\psi(\mathbf{u}):= \begin{cases}\int_{\Omega} \eta\left(\varphi_{0}\right) D \mathbf{u}: D \mathbf{u} d x & \text { if } \mathbf{u} \in H_{0}^{1}(\Omega)^{d} \cap L_{\sigma}^{2}(\Omega)=\operatorname{dom}(\psi) \\ +\infty & \text { else. }\end{cases}
$$

We note $\psi(0)=0$ and $\mathbf{v}_{0}$ is in the $L^{2}$-closure of $\operatorname{dom}(\psi)$, i.e., in $L_{\sigma}^{2}(\Omega)$. Hence, it remains to show that $\psi$ is convex and lower-semi-continuous and that $A$ is the subdifferential of $\psi$. Then, we can apply Theorem 5. But the first two properties are obvious. Thus, it remains to show the subdifferential property, which is satisfied by Lemma 4 below. Hence, we are able to apply Theorem 5 which yields the existence. Moreover, the initial condition is also fulfilled as Theorem 5 yields

$$
\mathbb{P}_{\sigma}\left(\rho_{0} \mathbf{v}(0)\right)=\mathcal{B} \mathbf{v}(0)=\mathcal{B} \mathbf{v}_{0}=\mathbb{P}_{\sigma}\left(\rho_{0} \mathbf{v}_{0}\right) \quad \text { in } L^{2}(\Omega)
$$

In particular, we can conclude

$$
0=\int_{\Omega} \mathbb{P}_{\sigma}\left(\rho_{0} \mathbf{v}(0)-\rho_{0} \mathbf{v}_{0}\right) \cdot \boldsymbol{\psi} \mathrm{d} x=\int_{\Omega}\left(\rho_{0} \mathbf{v}(0)-\rho_{0} \mathbf{v}_{0}\right) \cdot \boldsymbol{\psi} \mathrm{d} x
$$

for every $\psi \in C_{0, \sigma}^{\infty}(\Omega)$. By approximation, this identity also holds for $\psi:=\mathbf{v}(0)-$ $\mathbf{v}_{0} \in L_{\sigma}^{2}(\Omega)$ and we get

$$
\int_{\Omega} \rho_{0}\left|\mathbf{v}(0)-\mathbf{v}_{0}\right|^{2} \mathrm{~d} x=0 .
$$

This implies $\mathbf{v}(0)=\mathbf{v}_{0}$ in $L_{\sigma}^{2}(\Omega)$.

For the uniqueness, we consider $\mathbf{v}_{1}, \mathbf{v}_{2} \in W_{2}^{1}\left(0, T ; L_{\sigma}^{2}(\Omega)\right) \cap L^{\infty}\left(0, T ; H_{0}^{1}(\Omega) \cap\right.$ $\left.L_{\sigma}^{2}(\Omega)\right)$ such that (26) holds for a.e. $(t, x) \in(0, T) \times \Omega$. Then, $\mathbf{v}:=\mathbf{v}_{1}-\mathbf{v}_{2}$ solves the homogeneous equation a.e. in $(0, T) \times \Omega$. Testing this homogeneous equation with v, we get

$$
\int_{\Omega} \frac{1}{2} \rho_{0} \mathbf{v}_{\mid t=T}^{2} \mathrm{~d} x+\int_{0}^{T} \int_{\Omega} 2 \eta\left(\varphi_{0}\right) D \mathbf{v}: D \mathbf{v} \mathrm{d} x \mathrm{~d} t=0
$$

Hence, it follows $\mathbf{v} \equiv 0$ and therefore, $\mathbf{v}_{1}=\mathbf{v}_{2}$, which yields the uniqueness.

In the proof above, we used that the mapping $A$ coincides with the subdifferential $\partial \varphi$. More precisely, we have the following lemma.

Lemma 4. Let $\Omega \subseteq \mathbb{R}^{d}, d=2,3$, be a domain and $\psi: L_{\sigma}^{2}(\Omega) \rightarrow[0,+\infty]$ be given as in (31). Moreover, we consider $A: L_{\sigma}^{2}(\Omega) \rightarrow L_{\sigma}^{2}(\Omega)$ to be given as in (30). Then, it holds 
1. $\mathcal{D}(\partial \psi)=\mathcal{D}(A)$.

2. $\partial \psi(\boldsymbol{u})=\{$ A $\boldsymbol{u}\}$ for all $\boldsymbol{u} \in \mathcal{D}(A)$.

Proof. Remember that

$$
\mathcal{D}(\partial \psi)=\left\{\mathbf{v} \in L_{\sigma}^{2}(\Omega): \partial \psi(\mathbf{v}) \neq \emptyset\right\}
$$

and $\mathcal{D}(A)=H^{2}(\Omega)^{d} \cap H_{0}^{1}(\Omega)^{d} \cap L_{\sigma}^{2}(\Omega)$ by definition.

$\mathbf{1}^{\text {st }}$ part: $\mathcal{D}(A) \subseteq \mathcal{D}(\partial \psi)$ and $A \mathbf{u} \in \partial \psi(\mathbf{u})$ for every $\mathbf{u} \in \mathcal{D}(A)$.

To show the first part of the proof, let $\mathbf{u} \in \mathcal{D}(A)$ be given. If it holds $\mathbf{v} \in L_{\sigma}^{2}(\Omega)$ but $\mathbf{v} \notin H_{0}^{1}(\Omega)^{d}$, then the inequality

$$
\langle A \mathbf{u}, \mathbf{v}-\mathbf{u}\rangle_{L^{2}(\Omega)} \leq \psi(\mathbf{v})-\psi(\mathbf{u})
$$

is satisfied since it holds $\psi(\mathbf{v})=+\infty$ in this case by definition.

So, let $\mathbf{v} \in H_{0}^{1}(\Omega)^{d} \cap L_{\sigma}^{2}(\Omega)$. Then, it holds

$$
\begin{aligned}
\langle A \mathbf{u}, \mathbf{v}-\mathbf{u}\rangle_{L^{2}(\Omega)} & =-\int_{\Omega} \mathbb{P}_{\sigma} \operatorname{div}\left(2 \eta\left(\varphi_{0}\right) D \mathbf{u}\right) \cdot(\mathbf{v}-\mathbf{u}) \mathrm{d} x \\
& =\int_{\Omega} 2 \eta\left(\varphi_{0}\right) D \mathbf{u}: D \mathbf{v d} x-\int_{\Omega} 2 \eta\left(\varphi_{0}\right) D \mathbf{u}: D \mathbf{u d} x \\
& \leq \int_{\Omega} \eta\left(\varphi_{0}\right)|D \mathbf{u}|^{2} \mathrm{~d} x+\int_{\Omega} \eta\left(\varphi_{0}\right)|D \mathbf{v}|^{2} \mathrm{~d} x-2 \int_{\Omega} \eta\left(\varphi_{0}\right)|D \mathbf{u}|^{2} \mathrm{~d} x \\
& =\psi(\mathbf{v})-\psi(\mathbf{u})
\end{aligned}
$$

for every $\mathbf{v} \in H_{0}^{1}(\Omega)^{d} \cap L_{\sigma}^{2}(\Omega)$. This implies that $A \mathbf{u}$ is a subgradient of $\psi$ at $\mathbf{u}$, i.e., $A \mathbf{u} \in \partial \psi(\mathbf{u})$, and $\partial \psi(\mathbf{u}) \neq \emptyset$, i.e., $\mathbf{u} \in \mathcal{D}(A) \subseteq \mathcal{D}(\partial \psi)$. Hence, we have shown the first part of the proof.

$2^{\text {nd }}$ part: $\mathcal{D}(\partial \psi) \subseteq \mathcal{D}(A)$ and $\partial \psi(\mathbf{u})=\{A \mathbf{u}\}$.

Let $\mathbf{u} \in \mathcal{D}(\partial \psi) \subseteq \operatorname{dom}(\psi) \subseteq H_{0}^{1}(\Omega)^{d} \cap L_{\sigma}^{2}(\Omega)$ be given. Then, by definition of the subgradient there exists $\mathbf{w} \in \partial \psi(\mathbf{u}) \subseteq L_{\sigma}^{2}(\Omega)$ such that

$$
\psi(\mathbf{u})-\psi(\mathbf{v}) \leq\langle\mathbf{w}, \mathbf{u}-\mathbf{v}\rangle_{L^{2}(\Omega)}
$$

for every $\mathbf{v} \in L_{\sigma}^{2}(\Omega)$. Now, we choose $\mathbf{v}:=\mathbf{u}+t \tilde{\mathbf{w}}$ for some $\tilde{\mathbf{w}} \in H_{0}^{1}(\Omega)^{d} \cap L_{\sigma}^{2}(\Omega)$ and $t>0$. Then, inequality (32) yields

$$
\begin{aligned}
\psi(\mathbf{u})-\psi(\mathbf{v}) & =\int_{\Omega} \eta\left(\varphi_{0}\right) D \mathbf{u}: D \mathbf{u} \mathrm{d} x-\int_{\Omega} \eta\left(\varphi_{0}\right) D(\mathbf{u}+t \tilde{\mathbf{w}}): D(\mathbf{u}+t \tilde{\mathbf{w}}) \mathrm{d} x \\
& =-2 t \int_{\Omega} \eta\left(\varphi_{0}\right) D \mathbf{u}: D \tilde{\mathbf{w}} \mathrm{d} x-t^{2} \int_{\Omega} \eta\left(\varphi_{0}\right) D \mathbf{u}: D \tilde{\mathbf{w}} \mathrm{d} x \\
& \leq-t \int_{\Omega} \mathbf{w} \cdot \tilde{\mathbf{w}} \mathrm{d} x .
\end{aligned}
$$

Dividing this inequality by $-t<0$ and passing to the limit $t \searrow 0$ yields

$$
\int_{\Omega} \mathbf{w} \cdot \tilde{\mathbf{w}} \mathrm{d} x \leq \int_{\Omega} 2 \eta\left(\varphi_{0}\right) D \mathbf{u}: D \tilde{\mathbf{w}} \mathrm{d} x .
$$


When we replace $\tilde{\mathbf{w}}$ by $-\tilde{\mathbf{w}}$, we can conclude

$$
\int_{\Omega} \mathbf{w} \cdot \tilde{\mathbf{w}} \mathrm{d} x \geq \int_{\Omega} 2 \eta\left(\varphi_{0}\right) D \mathbf{u}: D \tilde{\mathbf{w}} \mathrm{d} x .
$$

Thus, it follows

$$
\int_{\Omega} \mathbf{w} \cdot \tilde{\mathbf{w}} \mathrm{d} x=\int_{\Omega} 2 \eta\left(\varphi_{0}\right) D \mathbf{u}: D \tilde{\mathbf{w}} \mathrm{d} x
$$

for every $\tilde{\mathbf{w}} \in H_{0}^{1}(\Omega)^{d} \cap L_{\sigma}^{2}(\Omega)$. Since we assumed $\mathbf{w} \in L_{\sigma}^{2}(\Omega)$, we can apply Lemma 5 below which yields $\mathbf{u} \in H^{2}(\Omega)^{d} \cap H_{0}^{1}(\Omega)^{d} \cap L_{\sigma}^{2}(\Omega)$. Using this regularity in (33), we can conclude

$$
\int_{\Omega} \mathbf{w} \cdot \tilde{\mathbf{w}} \mathrm{d} x=\int_{\Omega} 2 \eta\left(\varphi_{0}\right) D \mathbf{u}: D \tilde{\mathbf{w}} \mathrm{d} x=-\int_{\Omega} \mathbb{P}_{\sigma} \operatorname{div}\left(2 \eta\left(\varphi_{0}\right) D \mathbf{u}\right) \cdot \tilde{\mathbf{w}} \mathrm{d} x
$$

for every $\tilde{\mathbf{w}} \in H_{0}^{1}(\Omega)^{d} \cap L_{\sigma}^{2}(\Omega)$. Therefore, we obtain $\mathbf{w}=-\mathbb{P}_{\sigma} \operatorname{div}\left(2 \eta\left(\varphi_{0}\right) D \mathbf{u}\right)=$ $A \mathbf{u}$ in $L^{2}(\Omega)$, i.e., $\mathbf{u} \in \mathcal{D}(A)$ and $\partial \psi(\mathbf{u})=\{A \mathbf{u}\}$.

For the regularity of the Stokes system with variable viscosity, we used the following lemma.

Lemma 5. Let $\eta \in C^{2}(\mathbb{R})$ be such that $\eta(s) \geq s_{0}>0$ for all $s \in \mathbb{R}$ and some $s_{0}>0$, $\varphi_{0} \in W_{r}^{1}(\Omega), r>d \geq 2$, with $\left\|\varphi_{0}\right\|_{W_{r}^{1}(\Omega)} \leq R$, and let $\mathbf{u} \in H_{0}^{1}(\Omega)^{d} \cap L_{\sigma}^{2}(\Omega)$ be a solution of

$$
\left\langle 2 \eta\left(\varphi_{0}\right) D \mathbf{u}, D \tilde{\boldsymbol{w}}\right\rangle_{L^{2}(\Omega)}=\langle\mathbf{w}, \tilde{\boldsymbol{w}}\rangle_{L^{2}(\Omega)} \quad \text { for all } \tilde{\boldsymbol{w}} \in C_{0, \sigma}^{\infty}(\Omega)
$$

where $\mathbf{w} \in L^{2}(\Omega)^{d}$. Then, it holds $\mathbf{u} \in H^{2}(\Omega)^{d}$ and

$$
\|\mathbf{u}\|_{H^{2}(\Omega)} \leq C(R)\|\mathbf{w}\|_{L^{2}(\Omega)},
$$

where $C(R)$ only depends on $\Omega, \eta, r>d$, and $R>0$.

The proof can be found in [2, Lemma 4].

Lemma 3 implies $\mathbf{v} \in W_{2}^{1}\left(0, T ; L_{\sigma}^{2}(\Omega)\right) \cap L^{\infty}\left(0, T ; H_{0}^{1}(\Omega)^{d}\right)$. But as we want to show that $\mathbf{v}$ is in $X_{T}^{1}$, it remains to show $\mathbf{v} \in L^{2}\left(0, T ; H^{2}(\Omega)^{d}\right)$. To this end, we also use Lemma 5 above.

Lemma 6. For the unique solution $\mathbf{v} \in W_{2}^{1}\left(0, T ; L_{\sigma}^{2}(\Omega)\right) \cap L^{\infty}\left(0, T ; H_{0}^{1}(\Omega)^{d}\right)$ of (26)-(29) from Lemma 3, it holds $\mathbf{v} \in L^{2}\left(0, T ; H^{2}(\Omega)^{d}\right)$.

Proof. Let $\mathbf{v} \in W_{2}^{1}\left(0, T ; L_{\sigma}^{2}(\Omega)\right) \cap L^{\infty}\left(0, T ; H_{0}^{1}(\Omega)^{d}\right)$ be the unique solution of (26) from Lemma 3, i.e.,

$$
A(\mathbf{v}(t))=\mathbf{f}(t)-\frac{\mathrm{d}}{\mathrm{d} t}(\mathcal{B} \mathbf{v}(t))=\mathbf{f}(t)-\mathbb{P}_{\sigma}\left(\rho_{0} \partial_{t} \mathbf{v}(t)\right) \text { for all } 0<t<T
$$


Since the right-hand side is not the empty set, we get by definition of $A$

$$
\mathbb{P}_{\sigma}\left(\operatorname{div}\left(2 \eta\left(\varphi_{0}\right) D \mathbf{v}(t)\right)\right)=\mathbb{P}_{\sigma}\left(\rho_{0} \partial_{t} \mathbf{v}(t)\right)-\mathbf{f}(t) \text { for all } 0<t<T
$$

for given $\mathbf{f} \in L^{2}\left(0, T ; L_{\sigma}^{2}(\Omega)\right)$. From $\partial_{t} \mathbf{v} \in L^{2}\left(0, T ; L_{\sigma}^{2}(\Omega)\right)$, it follows

$$
\left\langle 2 \eta\left(\varphi_{0}\right) D \mathbf{v}(t), D \mathbf{w}\right\rangle_{L^{2}(\Omega)}=\left\langle\rho_{0} \partial_{t} \mathbf{v}(t)-\mathbf{f}(t), \mathbf{w}\right\rangle \quad \text { for every } \mathbf{w} \in C_{0, \sigma}^{\infty}(\Omega)
$$

and a.e. $t \in(0, T)$. Hence, we can apply Lemma 5 and obtain

$$
\begin{aligned}
\|\mathbf{v}(t)\|_{H^{2}(\Omega)} & \leq C(R)\left\|\rho_{0} \partial_{t} \mathbf{v}(t)-\mathbf{f}(t)\right\|_{L^{2}(\Omega)} \\
& \leq C(R)\left(\left\|\rho_{0} \partial_{t} \mathbf{v}(t)\right\|_{L^{2}(\Omega)}+\|\mathbf{f}(t)\|_{L^{2}(\Omega)}\right)
\end{aligned}
$$

for a.e. $t \in(0, T)$. Since the right-hand side of this inequality is bounded in $L^{2}(0, T)$, this shows the lemma.

We still need to ensure that $\left\|\mathcal{L}^{-1}\right\|_{\mathcal{L}\left(Y_{T}, X_{T}\right)}$ remains bounded. This is shown in the next lemma.

Lemma 7. Let the assumptions of Lemma 3 hold and $0<T_{0}<\infty$ be given. Then,

$$
\left\|\mathcal{L}_{1, T}^{-1}\right\|_{\mathcal{L}\left(Y_{T}^{1}, X_{T}^{1}\right)} \leq\left\|\mathcal{L}_{1, T_{0}}^{-1}\right\|_{\mathcal{L}\left(Y_{T_{0}}^{1}, X_{T_{0}}^{1}\right)}<\infty \quad \text { for all } 0<T<T_{0} .
$$

Proof. Let $0<T<T_{0}$ be given. Lemma 3 together with Lemma 6 yields that the operator $\mathcal{L}_{1, T}: X_{T} \rightarrow Y_{T}$ is invertible for every $0<T<\infty$ and every given $\mathbf{f} \in L^{2}\left(0, T ; L_{\sigma}^{2}(\Omega)\right), \varphi_{0} \in W_{r}^{1}(\Omega), \mathbf{v}_{0} \in H_{0}^{1}(\Omega)^{d} \cap L_{\sigma}^{2}(\Omega)$. Then, we define $\tilde{\mathbf{f}} \in L^{2}\left(0, T_{0}, L_{\sigma}^{2}(\Omega)\right)$ by

$$
\tilde{\mathbf{f}}(t):= \begin{cases}\mathbf{f}(t) & \text { if } t \in(0, T], \\ 0 & \text { if } t \in\left(T, T_{0}\right) .\end{cases}
$$

Due to Lemma 3 together with Lemma 6 , there exists a unique solution $\tilde{\mathbf{v}} \in X_{T_{0}}^{1}$ of

$$
\begin{array}{rlrl}
\mathbb{P}_{\sigma}\left(\rho_{0} \partial_{t} \tilde{\mathbf{v}}\right)-\mathbb{P}_{\sigma}\left(\operatorname{div}\left(2 \eta\left(\varphi_{0}\right) D \tilde{\mathbf{v}}\right)\right) & =\tilde{\mathbf{f}} & & \text { in } Q_{T_{0}}, \\
\operatorname{div}(\tilde{\mathbf{v}})=0 & & \text { in } Q_{T_{0},} \\
\tilde{\mathbf{v}} \mid \partial \Omega & =0 & & \text { on }\left(0, T_{0}\right) \times \partial \Omega, \\
\tilde{\mathbf{v}}(0) & =\mathbf{v}_{0} & & \text { in } \Omega .
\end{array}
$$

So let $\mathbf{v} \in X_{T}^{1}$ be the solution of the previous equations with $T_{0}$ replaced by $T$. Then, $\tilde{\mathbf{v}}$ and $\mathbf{v}$ solve these equations on $(0, T) \times \Omega$. Since the solution is unique, we can deduce $\tilde{\mathbf{v}}_{(0, T)}=\mathbf{v}$. Hence,

$$
\begin{aligned}
\left\|\mathcal{L}_{1, T}^{-1}(\mathbf{f})\right\|_{X_{T}^{1}} & =\|\mathbf{v}\|_{X_{T}^{1}} \leq\|\tilde{\mathbf{v}}\|_{X_{T_{0}}^{1}}=\left\|\mathcal{L}_{1, T_{0}}^{-1}(\tilde{\mathbf{f}})\right\|_{X_{T_{0}}^{1}} \\
& \leq\left\|\mathcal{L}_{1, T_{0}}^{-1}\right\|_{\mathcal{L}\left(Y_{T_{0}}^{1}, X_{T_{0}}^{1}\right)}\|\tilde{\mathbf{f}}\|_{Y_{T_{0}}^{1}}=\left\|\mathcal{L}_{1, T_{0}}^{-1}\right\|\left\|_{\mathcal{L}\left(Y_{T_{0}}^{1}, X_{T_{0}}^{1}\right)}\right\| \mathbf{f} \|_{Y_{T}^{1}},
\end{aligned}
$$

which shows the statement since it holds $\left\|\mathcal{L}_{1, T_{0}}^{-1}\right\|_{\mathcal{L}\left(Y_{T_{0}}^{1}, X_{T_{0}}^{1}\right)}<\infty$ by the bounded inverse theorem. 
Finally, we have to show invertibility of the second part of $\mathcal{L}$.

Lemma 8. Let Assumption 1 hold and $\varphi_{0} \in\left(L^{p}(\Omega), W_{p, N}^{4}(\Omega)\right)_{1-\frac{1}{p}, p}, f \in$ $L^{p}\left(0, T ; L^{p}(\Omega)\right)$ with $4<p<6$ be given. Then, for every $0<T<\infty$ there exists a unique

$$
\varphi \in L^{p}\left(0, T ; W_{p, N}^{4}(\Omega)\right) \cap\left\{u \in W_{p}^{1}\left(0, T ; L^{p}(\Omega)\right): u_{\mid t=0}=\varphi_{0}\right\}
$$

such that

$$
\begin{array}{rlrl}
\partial_{t} \varphi+\varepsilon m\left(\varphi_{0}\right) \Delta^{2} \varphi & =f & \text { in }(0, T) \times \Omega, \\
\partial_{n} \varphi \mid \partial \Omega & =0 & \text { on }(0, T) \times \partial \Omega, \\
\partial_{n} \Delta \varphi_{\mid \partial \Omega} & =0 & & \text { on }(0, T) \times \partial \Omega, \\
\varphi(0) & =\varphi_{0} & \text { in }\{0\} \times \Omega .
\end{array}
$$

Proof. The result follows from standard results on maximal regularity of parabolic equations, e.g., from [9, Theorem 8.2].

Analogously, to the previous part, we need to ensure that $\left\|\mathcal{L}^{-1}\right\|_{\mathcal{L}\left(Y_{T}, X_{T}\right)}$ remains bounded.

Lemma 9. Let the assumptions of Lemma 8 hold and $0<T_{0}<\infty$ be given. Then,

$$
\left\|\mathcal{L}_{2, T}^{-1}\right\|_{\mathcal{L}\left(Y_{T}^{2}, X_{T}^{2}\right)} \leq\left\|\mathcal{L}_{2, T_{0}}^{-1}\right\|_{\mathcal{L}\left(Y_{T_{0}}^{2}, X_{T_{0}}^{2}\right)}<\infty \quad \text { for all } 0<T<T_{0} .
$$

This lemma can be proven analogously to Lemma 7.

From the results of this section, Theorem 4 follows immediately.

Funding Open Access funding enabled and organized by Projekt DEAL.

Open Access. This article is licensed under a Creative Commons Attribution 4.0 International License, which permits use, sharing, adaptation, distribution and reproduction in any medium or format, as long as you give appropriate credit to the original author(s) and the source, provide a link to the Creative Commons licence, and indicate if changes were made. The images or other third party material in this article are included in the article's Creative Commons licence, unless indicated otherwise in a credit line to the material. If material is not included in the article's Creative Commons licence and your intended use is not permitted by statutory regulation or exceeds the permitted use, you will need to obtain permission directly from the copyright holder. To view a copy of this licence, visit http://creativecommons.org/licenses/ by/4.0/.

Publisher's Note Springer Nature remains neutral with regard to jurisdictional claims in published maps and institutional affiliations.

\section{REFERENCES}

[1] Abels, H.: Existence of weak solutions for a diffuse interface model for viscous, incompressible fluids with general densities. Comm. Math. Phys. 289(1), 45-73 (2009). https://doi.org/10.1007/ s00220-009-0806-4

[2] Abels, H.: On a diffuse interface model for two-phase flows of viscous, incompressible fluids with matched densities. Arch. Rat. Mech. Anal. 194(2), 463-506 (2009) 
[3] Abels, H., Breit, D.: Weak solutions for a non-Newtonian diffuse interface model with different densities. Nonlinearity 29(11), 3426-3453 (2016). https://doi.org/10.1088/0951-7715/29/11/3426

[4] Abels, H., Depner, D., Garcke, H.: Existence of weak solutions for a diffuse interface model for twophase flows of incompressible fluids with different densities. J. Math. Fluid Mech. 15(3), 453-480 (2013). https://doi.org/10.1007/s00021-012-0118-x

[5] Abels, H., Depner, D., Garcke, H.: On an incompressible Navier-Stokes/Cahn-Hilliard system with degenerate mobility. Ann. Inst. H. Poincaré Anal. Non Linéaire 30(6), 1175-1190 (2013). https:// doi.org/10.1016/j.anihpc.2013.01.002

[6] Abels, H., Garcke, H., Grün, G.: Thermodynamically consistent, frame indifferent diffuse interface models for incompressible two-phase flows with different densities. Math. Models Methods Appl. Sci. 22(3), 1150,013, 40 (2012)

[7] Abels, H., Garcke, H., Weber, J.: Existence of weak solutions for a diffuse interface model for two-phase flow with surfactants. Commun. Pure Appl. Anal. 18(1), 195-225 (2019). https://doi. org/10.3934/cpaa.2019011

[8] Amann, H.: Linear and Quasilinear Parabolic Problems, Volume 1: Abstract Linear Theory. Birkhäuser, Basel - Boston - Berlin (1995)

[9] Denk, R., Hieber, M., Prüss, J.: R-boundedness, fourier multipliers and problems of elliptic and parabolic type. Mem. Amer. Math. Soc. 166(788) (2003)

[10] Frigeri, S.: Global existence of weak solutions for a nonlocal model for two-phase flows of incompressible fluids with unmatched densities. Math. Models Methods Appl. Sci. 26(10), 1955-1993 (2016). https://doi.org/10.1142/S0218202516500494

[11] Gal, C.G., Grasselli, M., Wu, H.: Global weak solutions to a diffuse interface model for incompressible two-phase flows with moving contact lines and different densities. Arch. Ration. Mech. Anal. 234(1), 1-56 (2019). https://doi.org/10.1007/s00205-019-01383-8

[12] Giorgini, A.: Well-posedness of the two-dimensional Abels-Garcke-Grün model for two-phase flows with unmatched densities. Preprint, arXiv:2006.13060 (2020)

[13] Grün, G.: On convergent schemes for diffuse interface models for two-phase flow of incompressible fluids with general mass densities. SIAM J. Numer. Anal. 51(6), 3036-3061 (2013). https://doi.org/ $10.1137 / 130908208$

[14] Runst, T., Sickel, W.: Sobolev spaces of fractional order, Nemytskij operators, and nonlinear partial differential equations, De Gruyter Series in Nonlinear Analysis and Applications, vol. 3. Walter de Gruyter \& Co., Berlin (1996). https://doi.org/10.1515/9783110812411

[15] Showalter, R.E.: Monotone Operators in Banach Space and Nonlinear Partial Differential Equations, Mathematical Surveys and Monographs, vol. 49. American Mathematical Society, Providence, RI (1997)

[16] Weber, J.T.: Analysis of diffuse interface models for two-phase flows with and without surfactants. Ph.D. thesis, University of Regensburg, urn:nbn:de:bvb:355-epub-342471 (2016)

\author{
Helmut Abels and Josef Weber \\ Fakultät für Mathematik \\ Universität Regensburg \\ 93040 Regensburg \\ Germany \\ E-mail:helmut.abels@ur.de
}

Accepted: 26 October 2020 